\title{
International genome-wide meta-analysis identifies new primary biliary cirrhosis risk loci and targetable pathogenic pathways
}

Heather J. Cordell', Younghun Han², George F. Mells ${ }^{3}$, Yafang $\mathrm{Li}^{2}$, Gideon M. Hirschfield ${ }^{4}$, Casey S. Greene ${ }^{5}$, Gang Xie ${ }^{6}$, Brian D. Juran7 ${ }^{7}$ Dakai Zhu², David C. Qian², James A.B. Floyd ${ }^{8,9}$, Katherine I. Morley ${ }^{8,10,}$ Daniele Prati ${ }^{11}$, Ana Lleo ${ }^{12}$, Daniele Cusi ${ }^{13,14}$, Canadian-US PBC Consortium\#, Italian PBC Genetics Study Group\#, UK-PBC Consortium\#, M. Eric Gershwin ${ }^{15}$, Carl A. Anderson ${ }^{8}$, Konstantinos N. Lazaridis ${ }^{7}$, Pietro Invernizzi ${ }^{12,15}$,

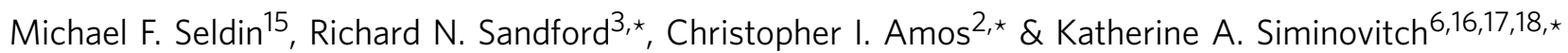

Primary biliary cirrhosis (PBC) is a classical autoimmune liver disease for which effective immunomodulatory therapy is lacking. Here we perform meta-analyses of discovery data sets from genome-wide association studies of European subjects ( $n=2,764$ cases and 10,475 controls) followed by validation genotyping in an independent cohort ( $n=3,716$ cases and 4,261 controls). We discover and validate six previously unknown risk loci for PBC $\left(P_{\text {combined }}<5 \times 10^{-8}\right)$ and used pathway analysis to identify JAK-STAT/IL12/IL27 signalling and cytokine-cytokine pathways, for which relevant therapies exist.

\footnotetext{
${ }^{1}$ Institute of Genetic Medicine, Newcastle University, Newcastle upon Tyne NE1 3BZ, UK. ${ }^{2}$ Center for Genomic Medicine, Department of Community and Family Medicine, Geisel School of Medicine, Dartmouth College, Lebanon, New Hampshire 03755, USA. ${ }^{3}$ Academic Department of Medical Genetics, Cambridge University, Cambridge CB2 OQQ, UK. ${ }^{4}$ Centre for Liver Research and NIHR Biomedical Research Unit, University of Birmingham, Birmingham B15 2TT, UK. ${ }^{5}$ Department of Genetics, Institute for Quantitative Biomedical Sciences, Geisel School of Medicine, Dartmouth College, Hanover, New Hampshire 03755, USA. ${ }^{6}$ Mount Sinai Hospital, Lunenfeld-Tanenbaum Research Institute and Toronto General Research Institute, Toronto, Ontario M5G 1X5, Canada. ${ }^{7}$ Center for Basic Research in Digestive Diseases, Mayo Clinic, Rochester, Minnesota 55905, USA. ${ }^{8}$ Wellcome Trust Sanger Institute, Wellcome Trust Genome Campus, Hinxton CB10 1SA, UK. ${ }^{9}$ William Harvey Research Institute, Queen Mary University of London, London EC1M 6BQ, UK. ${ }^{10}$ Institute of Psychiatry, Psychology \& Neuroscience, Kings College London, London SE5 8AF, UK. ${ }^{11}$ Department of Transfusion Medicine and Hematology, Ospedale Alessandro Manzoni, Lecco 23900, Italy. ${ }^{12}$ Center for Autoimmune Liver Diseases, IRCCS Instituto Clinico Humanitas, 20089 Rozzano, Italy. ${ }^{13}$ Università degli Studi di Milano, Milan 20129, Italy. ${ }^{14}$ Istituto di Tecnologie Biomediche Consiglio Nazionale delle Ricerche, Via Fratelli Cervi, 93,20090 Segrate, Milan, Italy. ${ }^{15}$ University of California - Davis, Davis, California 95616, USA. ${ }^{16}$ Department of Medicine, University of Toronto, Toronto, Ontario, Canada M5G 1 X5. ${ }^{17}$ Department of Immunology, University of Toronto, Toronto, Ontario, Canada M5G 1X5. ${ }^{18}$ Department of Molecular Genetics, University of Toronto, Toronto, Ontario, Canada M5G 1X5. * These authors contributed equally to this work. ${ }^{\#}$ A full list of consortium members appears at the end of the paper. Correspondence and requests for materials should be addressed to H.J.C. (email: heather.cordell@ncl.ac.uk) or K.A.S. (ksimin@mshri.on.ca).
} 
P rimary biliary cirrhosis (PBC) is a rare cholestatic liver disease characterized by progressive autoimmune destruction of intrahepatic bile ducts, leading to cirrhosis and liver failure in a substantial proportion of cases ${ }^{1}$. To date, four genome-wide association studies (GWAS) and two Illumina immunochip studies of PBC have confirmed associations at the human leukocyte antigen (HLA) locus and identified 27 non-HLA risk loci ${ }^{2-8}$. Consistent with GWAS data for other autoimmune diseases, results of these studies implicate immune-related genes in disease pathogenesis, but in general fail to pinpoint the disease-causal variants within the identified risk loci. To identify risk alleles that may be relevant to disease biology and treatment and illuminate additional PBC risk loci, we undertook a genome-wide meta-analysis (GWMA) combining North American, Italian and UK PBC GWAS data sets ${ }^{2,4,5}$. Functional annotation of the risk loci and pathway analyses were then performed to identify the alleles and pathways most relevant to disease cause and treatment.

\section{Results}

Discovery of new PBC risk loci. Following quality control, the combined discovery data set for GWMA consisted of 1,143,634 genotyped or imputed single-nucleotide polymorphisms (SNPs) in 2,764 cases and 10,475 controls (Supplementary Table 1). After genomic control correction and exclusion of known PBC risk loci from the final set of results, the inflation factor was $\lambda=1.043$ (Supplementary Fig. 1). Meta-analysis of this data set identified 23 loci at genome-wide level of significance $\left(P<5 \times 10^{-8}\right.$, calculated using logistic regression of individual discovery data sets in ProbABEL followed by genomic control correction of individual discovery data sets in $\mathrm{R}$ and fixed-effects meta-analysis in META, see Methods). Of these, 22 had been detected in previous studies and the 23rd corresponded to a most-likely spurious signal from a single imputed SNP on chromosome 13 (Supplementary Fig. 2, Supplementary Table 2). However, we found suggestive evidence of association $\left(P<2 \times 10^{-5}\right.$ from fixed-effects meta-analysis in META) at 41 loci not previously known to be associated with PBC. The top-scoring SNPs (or close proxies in strong linkage disequilibrium with the top-scoring SNP) from these and nine other loci (including the likely spurious chromosome 13 signal) were taken forward for genotyping in an independent panel consisting of 3,716 PBC cases and 4,261 controls. In total, 120 SNPs at 50 independent loci were taken forward for validation, of which 114 were successfully genotyped (Supplementary Data 1).

In the validation analysis, we confirmed association with SNPs at six loci not previously known to be associated with $\mathrm{PBC}$ $\left(P<4.4 \times 10^{-4}\right.$, equivalent to $P=0.05$ with a Bonferroni correction for 114 tests, calculated using logistic regression analysis of individual validation data sets in PLINK followed by meta-analysis in META, see Methods); meta-analysis of discovery and validation cohorts at these loci reached genome-wide levels of significance $\left(P_{\text {combined }}<5 \times 10^{-8}\right.$ from fixed-effect metaanalysis in META) (Table 1, Supplementary Figs 3 and 4). Furthermore, SNPs at two additional loci achieved $P$ values suggestive of association $\left(P<1 \times 10^{-3}\right.$ from fixed-effect metaanalysis in META, equivalent to $P=0.05$ with a Bonferroni correction for testing at 50 independent loci; Table 1, Supplementary Fig. 5). Newly identified PBC risk loci overlap with those of other autoimmune disorders and harbour several immunologically relevant candidate genes, most notably chemokine ligand 20 (CCL20) and interleukin 12B (IL12B; Table 1).

Discovery of candidate causal disease variants. In functional annotation of risk loci, we identified 199 candidate variants across
28 non-HLA risk loci with probabilistic identification of causal SNPs (PICS) probability $>0.0275$ (ref. 9). At each risk locus, the most-likely causal variant was the index variant, with median PICS probability of 0.224 and values up to 0.998 for rs 2546890 at 5q33.3 (Supplementary Data 2). Looking at all candidate variants across all risk loci, the majority were intronic, upstream or downstream gene variants with no predicted functional consequence $(99 / 199,40 \%)$. However, a substantial proportion (59/199, 30\%) were regulatory region variants, defined as SNPs located within regulatory features, including enhancers, promoters, transcription factor-binding sites and open chromatin regions (Supplementary Data 3). Notably, candidate variants at $18(64 \%)$ of the 28 annotated risk loci included at least one regulatory region variant. In contrast, only 5 of 199 candidates were missense variants (2.5\%) (Supplementary Table 3a). However, these included rs2297067 in EXOC3L4 at 14q32.32 and rs2304256 in TYK2 at 19p13.2, both predicted by SIFT and/or PolyPhen to be deleterious or potentially damaging ${ }^{10,11}$. Candidate variants included a single splice region variant, that is, rs17641524 at 1q31.3 that is predicted to affect splicing of DENND1B (Supplementary Table 3b).

We found that candidate variants at several risk loci are methylation quantitative trait loci (mQTLs), including mQTLs for DENNDIB, PLCL2, IRF5 and TNFRSF1A, all genes that are implicated in risk for other autoimmune diseases (Supplementary Data 4). We also found that candidate variants at several risk loci are expression quantitative trait loci (eQTLs) in lymphoblastoid and other cell lineages, including eQTLs for CCL20, IL12A, IRF5 and TYK2 (Supplementary Data 5).

At many risk loci, functional annotation highlighted a single candidate gene (Supplementary Data 2). However, most risk loci contained multiple compelling candidate variants. This complexity is well illustrated by the composite of candidate variants at the PLCL2 gene and MANBA gene loci, which include multiple eQTL and mQTL SNPs. Thus, despite the presence of many candidate variants with regulatory or epigenetic roles within PBC risk loci, more direct biological experimental approaches are required to pinpoint the disease-causal variants at these loci.

We also applied functional GWAS (FGWAS) and its associated annotation file $e^{12}$ to our full set of discovery GWMA results and thereby identified 75 annotations with enrichment $(P<0.01$ from FGWAS) of GWMA association signals (Supplementary Data 6). After a stepwise selection approach similar to that of Pickrell $^{12}$, the best-fitting model included six annotations highlighting negative enrichment of repressed chromatin regions in a lymphoblastoid cell line, and positive enrichment of DNase-I-hypersensitive sites in a variety of cell types, in particular CD20 + and Th1 T cells (Supplementary Table 4).

Identification of candidate targetable biological pathways. To identify biological pathways involved in development of PBC, we conducted pathway analysis using GCTA $^{13}$ followed by i-GSEA4GWAS ${ }^{14}$. We identified several immunoregulatory pathways associated with $\mathrm{PBC}$, in particular, IL-12 and other cytokines as well as T-cell signalling pathways. To account for bias that might result from the strong HLA association with PBC, we repeated this analysis with SNPs/genes in the HLA region excluded. Notably, IL-12, IL-27 and JAK-STAT signalling pathways were still associated with PBC, even after their HLA contribution had been removed (Table 2).

We identified molecules that targeted these pathways by overlaying the Drug Gene Interaction database ${ }^{15}$ and calculating a pathway specificity score and Jaccard index of each drug for each of the pathways that remained associated with PBC after the 
Table 1 | PBC risk loci identified in the current study.

\section{a. Confirmed risk loci (validation $P<4.4 \times 10^{-4}$ resulting in combined $P<5 \times 10^{-8}$ )}

\begin{tabular}{|c|c|c|c|c|c|c|c|c|c|c|}
\hline Locus & SNP & $\begin{array}{c}\text { Position } \\
\text { (build 38) }\end{array}$ & A1/A2 & $\begin{array}{c}\text { Discovery } \\
\mathbf{P} \\
\end{array}$ & $\begin{array}{c}\text { Validation } \\
\mathbf{P} \\
\end{array}$ & $\begin{array}{c}\text { Joint } \\
P\end{array}$ & OR $(95 \% \mathrm{Cl})$ & Region (build 38) & $\begin{array}{l}\text { Nearby genes and } \\
\text { functional annotation }\end{array}$ & $\begin{array}{l}\text { Autoimmune } \\
\text { overlap }\end{array}$ \\
\hline $2 q 12.1$ & rs12712133 & $102,249,813$ & $A / G$ & $1.62 \times 10^{-5}$ & $7.94 \times 10^{-5}$ & $5.19 \times 10^{-9}$ & $1.14(1.07-1.21)$ & $102,118,975-102,438,307$ & $\begin{array}{l}\text { IL1R1, IL1RL2 } \\
\text { FAM183DP, } \\
\text { IL181RL1 } \\
\text { IL18R1, LOC100422339, } \\
\text { IL18RAP, MIR4772 }\end{array}$ & $\mathrm{CD}, \mathrm{CeD}$ \\
\hline $2 q 36.3$ & rs4973341 & $227,795,646$ & $\mathrm{C} / \mathrm{T}$ & $6.48 \times 10^{-7}$ & $7.73 \times 10^{-5}$ & $2.34 \times 10^{-10}$ & $0.82(0.74-0.90)$ & $227,747,828-227,815,647$ & $\begin{array}{l}\text { RNA5SP121, SNRPGP8, } \\
\text { LOC100533842, } \\
\text { CCL20 }\end{array}$ & \\
\hline 4p16.3 & rs11724804 & 971,991 & $A / G$ & $3.67 \times 10^{-7}$ & $4.25 \times 10^{-6}$ & $9.01 \times 10^{-12}$ & $1.22(1.12-1.33)$ & $853,681-1,014,424$ & $\begin{array}{l}\text { GAK, TMEM175, } \\
\text { DGKQ } \\
\text { IDUA, }, \text { FGFRL1 }\end{array}$ & \\
\hline $5 q 21.1$ & rs526231 & $103,345,680$ & $\mathrm{~T} / \mathrm{C}$ & $3.10 \times 10^{-5}$ & $9.39 \times 10^{-5}$ & $1.14 \times 10^{-8}$ & $0.87(0.81-0.93)$ & $102,939,698-103,416,571$ & $\begin{array}{l}\text { PAM } \$, E I F 3 K P 1, \text { GIN1, } \\
\text { PPIP5K2, C5orf30 }\end{array}$ & RA \\
\hline $5 q 33.3$ & rs2546890 & $159,332,892$ & $G / A$ & $1.20 \times 10^{-6}$ & $1.89 \times 10^{-5}$ & $1.06 \times 10^{-10}$ & $0.87(0.82-0.93)$ & $159,117,927-159,414,310$ & $\begin{array}{l}\text { RNF145, } \overline{\text { UBLCP1, }} \\
\text { RNU4ATAC2P, IL12B, } \\
\text { LOC285626 }\end{array}$ & Pso, MS, CD \\
\hline $6 q 23.3$ & rs6933404 & $137,638,098$ & $\mathrm{C} / \mathrm{T}$ & $9.47 \times 10^{-7}$ & $2.84 \times 10^{-5}$ & $1.27 \times 10^{-10}$ & $1.18(1.09-1.27)$ & $137,571,557-137,803,754$ & $\begin{array}{l}\text { LOC102723649, } \\
\text { LOC442263, OLIG3 }^{\dagger}, \\
\text { TNFAIP3 }^{\dagger}\end{array}$ & $\begin{array}{l}\text { RA, SLE, SjS, } \\
\text { CeD, UC, MS }\end{array}$ \\
\hline
\end{tabular}

b. Suggestive risk loci (validation $P<1 \times 10^{-3}$ )

\begin{tabular}{|c|c|c|c|c|c|c|c|c|c|c|}
\hline Locus & SNP & $\begin{array}{c}\text { Position } \\
\text { (build 38) }\end{array}$ & A1/A2 & $\begin{array}{c}\text { Discovery } \\
P \\
\end{array}$ & $\begin{array}{c}\text { Validation } \\
P\end{array}$ & $\begin{array}{c}\text { Joint } \\
P\end{array}$ & $\begin{array}{l}\text { OR for } \mathrm{A1} \\
(95 \% \mathrm{Cl})\end{array}$ & Region (build 38) & $\begin{array}{l}\text { Nearby genes and } \\
\text { functional annotation }\end{array}$ & $\begin{array}{c}\text { Autoimmune } \\
\text { overlap }\end{array}$ \\
\hline $5 q 23.1$ & rs2434360 & $116,057,393$ & $T / G$ & $3.20 \times 10^{-3}$ & $9.94 \times 10^{-4}$ & $1.04 \times 10^{-5}$ & $1.14(1.05-1.23)$ & $116,032,882-116,163,459$ & $\begin{array}{l}\text { RPS25P6, ARL14EPL, } \\
\text { COMMD10 }\end{array}$ & \\
\hline 16p11 & rs1859308 & $27,386,677$ & $\mathrm{~T} / \mathrm{C}$ & $7.72 \times 10^{-5}$ & $5.37 \times 10^{-4}$ & $1.63 \times 10^{-7}$ & $0.85(0.77-0.93)$ & $27,359,133-27,434,733$ & $I L 4 R, I L 21 R$ & \\
\hline
\end{tabular}

$\mathrm{A} 1$, tested allele; $\mathrm{CD}$, Crohn disease; $\mathrm{CeD}$, coeliac disease; $\mathrm{Cl}$, confidence interval; MS, multiple sclerosis; OR, odds ratio in validation cohorts; Pso, psoriasis; RA, rheumatoid arthritis; SjS, Sjogren syndrome; SLE, systemic lupus erythematosus; SNP, single-nucleotide polymorphism; UC, ulcerative colitis.

PBC risk loci identified in the current study. SNPs were taken forward for validation based on having a discovery $P$ value $<2 \times 10^{-5}$ (or, in the case of rs 526231 and rs 2434360 , based on acting as proxy for a SNP with a $P$ value $<2 \times 10^{-5}$ ). Discovery $P$ values were calculated using logistic regression of individual discovery data sets in ProbABEL followed by genomic control correction of individual discovery data sets in R and fixed-effects meta-analysis in META; validation $P$ values were calculated using logistic regression of individual data sets in PLINK followed by fixed-effect meta-analysis in META; joint $P$ values were calculated using fixed-effect meta-analysis of discovery and validation data sets in META; see Methods. Autoimmune overlap refers to overlap between risk loci for PBC and those of other autoimmune conditions.

*Functional annotation.

†Regulatory variants: The index SNP or variants in strong linkage disequilibrium (LD, $\left.r^{2} \geq 0.8\right)$ with the index SNP at this locus overlap regulatory elements that are related to the annotated gene (Supplementary Table 3).

$\ddagger$ MQTLs: The index SNP or variants in strong LD are correlated to methylation related to the annotated gene (Supplementary Data 4)

§eQTLs: The index SNP or variants in strong LD are correlated to expression of the annotated gene (see Supplementary Data 3).

HLA contribution had been removed (Table 2, Supplementary Data 7). This combined analysis identified pathways and immunomodulatory agents that represent promising leads for further study in models of PBC.

\section{Discussion}

The current study adds to our knowledge of the genetic architecture of PBC. Notably, our data identify CCL2O as a candidate risk gene for PBC. Chemokine ligand 20 (CCL20) and its chemokine receptor CCR6 contribute to the formation and function of mucosal lymphoid tissues and are notably, in the context of the immune-mediated lymphocytic cholangitis characteristic of PBC, involved in the localization of Th17 cells and CD8 effector T cells to cholangiocytes and the periductal area in portal tracts ${ }^{16}$. This study also reinforces the importance of IL-12 and JAK-STAT signalling in this disease.

The functional annotation of risk loci has helped to assign priority to the candidate genes at newly identified and established risk loci. Furthermore, the identification of disease-associated regulatory variants at multiple risk loci emphasizes the potential importance of gene regulation in the pathogenesis of PBC (and presumably other complex disorders). This possibility is corroborated by the finding of numerous risk loci wherein the index and/or closely related SNPs that appear to represent regulatory, mQTL and/or eQTLs variants related to the nearby gene. Via the FGWAS analysis, this study also suggests particular importance of CD20 $+\mathrm{B}$ cells and Th1 cells in the pathogenesis of PBC. However, both the cell types and the specific gene variants most relevant to $\mathrm{PBC}$ require further investigation and in particular exploration of the tissue-specific functional effects of the disease-associated variants.

By looking for drug-gene interactions, we have identified candidate drugs targeting specific, PBC-associated pathways, creating new opportunities to re-purpose available drugs for targeted immune therapy. Despite the speculative nature of this analysis, the data provide a start point in the search for novel therapies that are urgently needed to improve outcomes for PBC patients.

\section{Methods}

Study samples and genotyping. The use of human subjects for this study was approved by the University Health Network Research Ethics Board, The Mayo Clinic Institutional Review Board, Etico Indipendente IRCCS Istituto Clinico Humanitas, UC Davis Institutional Review Board and the Oxford Research Ethics Committee.

All PBC cases included in the Canadian-US, Italian and UK discovery and validation cohorts fulfilled the American Association for the Study of Liver Diseases criteria for PBC

The Canadian-US discovery cohort included 499 PBC cases who were self-reported whites of European descent and 390 healthy Canadian controls, all genotyped using the Illumina HumanHap370 BeadChip. Additional controls included in this cohort were 1,094 control subjects provided from the Prostate Cancer Genetics Markers Susceptibility (CGEMS), 1,142 controls from the Breast CGEMS studies and 1,748 controls from the New York Cancer Project, all of whom who were genotyped on an Illumina $550 \mathrm{~K}$ bead array ${ }^{4}$. Following all quality control (QC) procedures, the final Canadian-US discovery set included 499 PBC cases and 4,374 controls.

The PBC cases included in the Italian discovery cohort were self-reported whites of Italian descent genotyped using the Illumina Human610-Quad BeadChip. Controls in this cohort were healthy Italians genotyped using the Illumina 1M-duo 
Table 2 | Results from pathway analysis in iGSEA4GWAS.

\section{Gene set}

NO2-dependent IL-12 pathway in NK cells

JAK-STAT signalling pathway ${ }^{*} \dagger$

IL-12 mediated signalling events

IL-12- and Stat4-dependent signalling in Th1 development ${ }^{\star}, \dagger$

Interferon signalling

PD-1 signalling

Phosphorylation of CD3 and TCR- $\zeta$ chains

IL-27-mediated signalling events ${ }^{\star \dagger}$

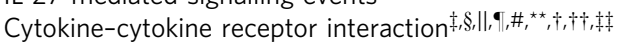

IFN- $\gamma$ signalling

MHC class II antigen presentation

Cytokine signalling in immune system

Antigen processing and presentation

Intestinal immune network for IgA production

Co-stimulation by the CD28 family

IL-2 mediated signalling events

TCR signalling

Downstream TCR signalling

Cell adhesion molecules

Th1, Th2 differentiation ${ }^{\dagger}$

$\mathrm{IL}-2$ receptor beta chain in T-cell activation

Interferon $\alpha / \beta$ signalling

IL-23-mediated signalling events
FDR (with HLA)

$6.7 \times 10^{-4}$

0.001

0.001

PID

Biocarta

REACTOME 0.001

REACTOME 0.001

REACTOME 0.001

PID 0.001

KEGG 0.002

REACTOME $\quad 0.002$

REACTOME 0.003

REACTOME $\quad 0.004$

KEGG 0.004

KEGG 0.004

REACTOME $\quad 0.005$

PID 0.008

REACTOME $\quad 0.008$

REACTOME 0.008

KEGG $\quad 0.015$

Biocarta $\quad 0.019$

Biocarta $\quad 0.021$

REACTOME 0.035

PID 0.039
FDR (without HLA)

0.013

$<0.001$

$<0.001$

0.010

0.012

FDR, false discovery rate; IFN, interferon; IL, interleukin; PD, programmed cell death; TCR, T cell antigen receptor; NK, natural killer.

Gene sets with FDR $<0.05$ are listed. Results are shown with or without inclusion in the analysis of SNPs within the HLA region. The top 10 hits from our drug-positioning analysis using a combined pathway from the HLA excluded set are indicated by symbols for the associated pathways that they affect.

${ }^{\star}$ Tofacitinib.

$\dagger$ Glatiramer acetate.

$\ddagger$ Axitinib.

§azopanib.

IIVatalanib.

- Cediranib.

\#X-82.

${ }^{\star \star}$ Telatinib.

†Linifanib.

$\ddagger$ Tandutinib.

array. Following QC procedures, the final Italian discovery set comprised 449 cases and 940 controls.

The PBC cases included in the UK discovery cohort were self-reported whites of British descent, genotyped using the Illumina Human-660 W Quad array. Controls in this cohort were 5,163 population controls genotyped on the Illumina $1 \mathrm{M}$-Duo array as part of the Wellcome Trust Case Control Consortium 2 project. Following QC procedures, the UK discovery set comprised 1,816 cases and 5,161 controls.

The 'Canadian' 903 PBC cases and 834 controls included in the validation studies were self-reported whites of European descent recruited from Canada, Europe and the United States to an ongoing PBC genetics study based in Toronto. The 721 'US' PBC cases and 294 controls included in the validation studies were self-reported whites of European descent enroled in the Mayo Clinic PBC Genetic Epidemiology registry and biorepository based at the Mayo Clinic in Rochester (https://clinicaltrials.gov/ct2/show/NCT01161953?term=pbc\&rank=5).The Italian PBC cases and controls included in the validation studies were self-reported whites of Italian descent recruited to the Italian PBC Genetics study based at Instituto Humanitas in Milan. The Italian controls were obtained from Ospedale Alessandro Manzoni, Lecco, Italy and were unrelated healthy volunteers with no known non-Italian heritage. Cases and controls from the Canadian, Italian and the US cohorts were genotyped at the University Health Network/Mount Sinai Hospital Clinical Genomics Centre using a Sequenom iPLEX Gold assay. Following QC procedures, the final validation set included 903 cases and 834 controls from Canada; 300 cases and 618 controls from Italy; and 721 cases and 294 controls from the United States (Supplementary Table 1).

The 'UK' $\mathrm{PBC}$ cases included in the validation studies were self-reported whites of British descent recruited to the UK-PBC project via the UK-PBC Consortium (http://www.uk-pbc.com/). Cases were genotyped using Sequenom iPLEX Gold assay at the Wellcome Trust Sanger Institute Genotyping Facility (http:// www.sanger.ac.uk/). The UK validation control data were obtained from the TwinsUK resource, an adult twin registry comprising 12,000 (predominantly female) British twins. Genotype data for 3,512 twin individuals (genotyped using the Illumina HumanHap610 array) were obtained from the Department of Twin Research and Genetic Epidemiology at King's College London. One twin from each genotyped pair was included in the current study, amounting to 2,603 unrelated individuals. Following QC procedures, the final UK validation set comprised 1,792 PBC cases and 2,515 TwinsUK controls (Supplementary Table 1).
Quality control. We implemented a standard QC pipeline across all three discovery data sets, over-and-above QC procedures carried out in the respective primary analyses $2,4,5$. QC checks were carried out using the software package PLINK $^{17}$. Within each discovery data set we removed SNPs with a genotype call rate $<95 \%$; minor allele frequency $<0.05$; significant deviation from Hardy Weinberg Equilibrium in controls $\left(P<10^{-5}\right)$ or a large difference $(>5 \%)$ in the proportion of missing genotypes in cases versus controls. We removed samples showing high rates of missing data $(>90 \%)$; whole-genome heterozygosity $>$ six s.d. from the mean; estimated proportion of identity by descent (IBD) sharing with another sample $>0.1$, or apparent gender discrepancies (based on X-chromosomal heterozygosity $>0.2$ for men and $<0.2$ for women). Principal component analysis (based on a subset of 32,000 highly informative SNPs) was carried out using the 'smartpca' routine of the EIGENSOFT package ${ }^{18}$ to identify population outliers for exclusion and to identify principal components that differed between cases and controls; these principal components were used as covariates in subsequent association analyses.

Genome-wide imputation. We used the SNPs and samples passing QC to carry out genome-wide imputation within each of our cohorts using the software package $\mathrm{MaCH}^{19}$ with HapMap3 CEU + TSI samples as reference data sets. Within each cohort we used approximately the same set of genotyped SNPs in cases and controls to ensure similar levels of informativity. Following imputation, we retained only those SNPs displaying minor allele frequency $>0.005$ and imputation quality score $R^{2}>0.5$ in all three cohorts.

Statistical analysis of discovery cohorts. Within each cohort we carried out association analysis of the genome-wide imputed data allowing for imputation uncertainty using the software package ProbABEL ${ }^{20}$. We performed logistic regression of disease phenotype on allele dosage; principal components that differed between cases and controls were included as covariates to help correct for population stratification. Quantile-quantile plots of the genome-wide set of test statistics were examined and genomic control correction was carried out within each cohort by multiplying the standard error of the estimated log odds ratio for each SNP by the square root of the genomic control inflation factor $\lambda$ (ref. 21). The resulting log odds ratios and adjusted standard errors from all three cohorts were 
meta-analysed using the software package META to produce the final set of genome-wide discovery results ${ }^{22}$.

Validation analysis. We selected loci for validation if they achieved suggestive level of significance in the discovery analysis (minimum $P<2 \times 10^{-5}$ ) and were not already known to be associated with PBC. We also selected loci for validation if they had achieved genome-wide significant association in one previous study but had never been validated in an independent cohort. We selected approximately two validation SNPs per locus; for loci displaying extended patterns of linkage disequilibrium or harbouring several putative independent association signals we attempted to select two validation SNPs within each subregion.

Within each locus chosen for validation we assigned priority to SNPs according to whether they had been genotyped in the TwinsUK cohort (which was used as a validation cohort for the UK validation cases). One SNP selected for validation (rs2297067) did not have genotype data available in TwinsUK and was therefore imputed within TwinsUK based on genotyped SNPs in the surrounding 5-Mb region using the software packages SHAPEIT ${ }^{23}$ and IMPUTE2 (ref. 24), with 1,000 Genomes (Phase I version 3 integrated data, released on March 2012) used as a reference sample. The TwinsUK cohort was subjected to a variety of additional QC checks as described previously ${ }^{25}$; the 2,515 controls used here correspond to the 2,520 controls used previously with an additional five exclusions due to discrepant gender ${ }^{25}$.

Within each validation cohort we carried out case/control association analysis of those SNPs that were successfully genotyped using logistic regression in PLINK. Results from the four validation cohorts (or from the combined discovery and validation cohorts) were combined via meta-analysis in META.

Imputation to 1,000 Genomes within validated loci. Imputation within the discovery cohorts was carried out at the six validated loci using the software packages SHAPEIT ${ }^{23}$ and IMPUTE2 (ref. 24) with the 1,000 Genomes (Phase I integrated variant set, release December and June 2013) used as a reference panel. The same genotyped SNPs that had been used to inform HapMap3 imputation for the discovery analysis were used for the 1,000 Genomes imputation within these targeted regions. Association analysis of SNPs passing post-imputation QC ('info' score $>0.5$ ) was carried out separately within each cohort, the results were genomic control corrected by multiplying the standard error of the estimated log odds ratio for each SNP by the square root of the previously estimated genomic control inflation factor $\lambda$ for each cohort, and results were combined across the cohorts via meta-analysis in META. This confirmed the findings from our original (HapMap3) imputation experiment but did not identify any substantially stronger associations or candidate causal variants than we had already found.

\section{Functional annotation of validated loci. Left and right boundaries for each} associated region were defined by finding a $0.1-\mathrm{cM}$ interval either side of the most strongly associated SNP where no SNP has $P<1 \times 10^{-5}$. We looked for overlap between $\mathrm{PBC}$ risk loci and confirmed risk loci for other autoimmune conditions using ImmunoBase, a web-based resource focused on the genetics and genomics of immunologically related human diseases (http://www.immunobase.org/). To assign priority to candidate genes and candidate variants at risk loci, we used the online PICS (Probabilistic Identification of Causal SNPs) algorithm to identify candidate variants at each risk locus with a PICS probability $>0.0275$ (http://www.broadinstitute.org/pubs/finemapping/?q=pics) ${ }^{9}$. We adopted this threshold to be consistent with Farh et al..$^{9}$ in their paper describing the approach. Given an index SNP corresponding to the most associated SNP in a locus, the PICS algorithm calculates (based on the known linkage disequilibrium pattern in the region, as measured in a large Immunochip or 1000 Genomes reference sample) a score for each SNP in the region, representing the extent to which that SNP could, in fact, be the true causal SNP, allowing for statistical sampling variation.

We then used the Ensembl Variant Effect Predictor web tool to annotate candidate variants for their predicted functional consequences (http:// www.ensembl.org/info/docs/tools/vep/index.html). We used Genevar to evaluate the measured effects of these variants on DNA methylation in tissue collected from 856 healthy female twins of the MuTHER resource (http://www.sanger.ac.uk/ resources/software/genevar/) 26,27 . We used Genevar ${ }^{26}$, seeQTL (http:// www.bios.unc.edu/research/genomic_software/seeQTL/) ${ }^{28}$ and the University of Chicago eQTL browser (http://eqtl.uchicago.edu/cgi-bin/gbrowse/eqtl/) to identify eQTLs amongst candidate variants.

We also used the FGWAS software and its associated annotation file (containing 450 genomic annotations of various types), applied to our full set of GWMA results, to investigate the extent to which genetic variants associated with $\mathrm{PBC}$ were enriched within specific annotation categories ${ }^{12}$. Testing each annotation individually, we found 75 annotations that showed enrichment $(P<0.01)$ of GWMA association signals; as many of these annotations are correlated with one another we used a stepwise selection approach followed by cross-validation to mitigate overfitting (similar to the procedure performed by Pickrell ${ }^{12}$ ) on these 75 annotations to identify a final best-fitting model that included 6 annotations. Annotation information used by FGWAS was derived from a variety of sources including Maurano et al. ${ }^{29}$, Thurman et al. ${ }^{30}$ and Hffman et al. ${ }^{31}$ (see Appendix of Pickrell ${ }^{12}$ for details).
Pathway analysis. Using summary results from the GWMA (effect size, standard error and allele frequency) along with SNP linkage disequilibrium estimated from the Italian GWAS individual-level genotype data, we performed approximate conditional analysis using the software GCTA ${ }^{13}$. Only the independently associated signals with conditional $P$ value and $P_{\mathrm{GWMA}}$ both $<0.001$ were retained for further consideration. We submitted the rsIDs and $P_{\mathrm{GWMA}}$ of these SNPs as well as gene sets from BioCarta, KEGG, PID and Reactome curated by MSigDB (as of 26 March 2014 ) to the i-GSEA4GWAS web server ${ }^{14}$. This programme identified genes within $20 \mathrm{~kb}$ of the SNPs and represented each gene by the greatest $-\log P_{\mathrm{GWMA}}$ of the SNP(s) mapped to it. Gene sets were then assessed for enrichment with significant genes while SNP label permutations were conducted to correct for bias from variations in gene size and gene set size. False discovery rate was used to correct for multiple testing based on the distributions of enrichment scores generated by permutation.

Drug-pathway analysis. To identify drugs that affected the pathways associated with PBC (when the HLA locus was excluded), we first identified the genes participating in each pathway. We then downloaded drug-gene associations from the Drug Gene Interaction database ${ }^{15}$ and scored each drug by the proportion of each its targets that were in each pathway, which we termed as the drug's pathway specificity. As a secondary scoring metric, we evaluated the proportion of each pathway affected by the drug using the Jaccard index on the respective sets of pathway genes and targeted genes. To identify promising drug candidates, we ranked drugs first by our primary specificity metric and then by the secondary Jaccard index.

\section{References}

1. Hirschfield, G. M. \& Gershwin, M. E. The immunobiology and pathophysiology of primary biliary cirrhosis. Annu. Rev. Pathol. 8, 303-330 (2013).

2. Hirschfield, G. M. et al. Primary biliary cirrhosis associated with HLA, IL12A and IL12RB2 variants. N. Engl. J. Med. 360, 2544-2555 (2009).

3. Hirschfield, G. M. et al. Variants at IRF5-TNPO3, 17q12-21 and MMEL1 are associated with primary biliary cirrhosis. Nat. Genet. 42, 655-657 (2010).

4. Liu, X. et al. Genome-wide meta-analyses identify three loci associated with primary biliary cirrhosis. Nat. Genet. 42, 658-660 (2010).

5. Mells, G. F. et al. Genome-wide association study identifies 12 new susceptibility loci for primary biliary cirrhosis. Nat. Genet. 43, 329-332 (2011)

6. Juran, B. D. et al. Immunochip analyses identify a novel risk locus for primary biliary cirrhosis at 13q14, multiple independent associations at four established risk loci and epistasis between $1 \mathrm{p} 31$ and $7 \mathrm{q} 32$ risk variants. Hum. Mol. Genet. 21, 5209-5221 (2012).

7. Liu, J. Z. et al. Dense fine-mapping study identifies new susceptibility loci for primary biliary cirrhosis. Nat. Genet. 44, 1137-1141 (2012).

8. Nakamura, M. et al. Genome-wide association study identifies TNFSF15 and POU2AF1 as susceptibility loci for primary biliary cirrhosis in the Japanese population. Am. J. Hum. Genet. 91, 721-728 (2012).

9. Farh, K. K. et al. Genetic and epigenetic fine mapping of causal autoimmune disease variants. Nature 518, 337-343 (2015).

10. Kumar, P., Henikoff, S. \& Ng, P. C. Predicting the effects of coding non-synonymous variants on protein function using the SIFT algorithm. Nat. Protoc. 4, 1073-1081 (2009).

11. Adzhubei, I. A. et al. A method and server for predicting damaging missense mutations. Nat. Methods 7, 248-249 (2010).

12. Pickrell, J. K. Joint analysis of functional genomic data and genome-wide association studies of 18 human traits. Am. J. Hum. Genet. 94, 559-573 (2014).

13. Yang, J., Lee, S. H., Goddard, M. E. \& Visscher, P. M. GCTA: a tool for genome-wide complex trait analysis. Am. J. Hum. Genet. 88, 76-82 (2011).

14. Zhang, K., Cui, S., Chang, S., Zhang, L. \& Wang, J. i-GSEA4GWAS: a web server for identification of pathways/gene sets associated with traits by applying an improved gene set enrichment analysis to genome-wide association study. Nucleic Acids Res. 38, W90-W95 (2010).

15. Griffith, M. et al. DGIdb: mining the druggable genome. Nat. Methods 10, 1209-1210 (2013).

16. Oo, Y. H. et al. CXCR3-dependent recruitment and CCR6-mediated positioning of Th-17 cells in the inflamed liver. J. Hepatol. 57, 1044-1051 (2012).

17. Purcell, S. et al. PLINK: a tool set for whole-genome association and population-based linkage analyses. Am. J. Hum. Genet. 81, 559-575 (2007).

18. Price, A. L. et al. Principal components analysis corrects for stratification in genome-wide association studies. Nat. Genet. 38, 904-909 (2006).

19. Li, Y., Willer, C. J., Ding, J., Scheet, P. \& Abecasis, G. R. MaCH: using sequence and genotype data to estimate haplotypes and unobserved genotypes. Genet. Epidemiol. 34, 816-834 (2010).

20. Aulchenko, Y. S., Struchalin, M. V. \& van Duijn, C. M. ProbABEL package for genome-wide association analysis of imputed data. BMC Bioinformatics 11, 134 (2010). 
21. Devlin, B. \& Roeder, K. Genomic control for association studies. Biometrics 55, 997-1004 (1999).

22. Liu, J. Z. et al. Meta-analysis and imputation refines the association of $15 \mathrm{q} 25$ with smoking quantity. Nat. Genet. 42, 436-440 (2010).

23. Delaneau, O., Marchini, J. \& Zagury, J. F. A linear complexity phasing method for thousands of genomes. Nat. Methods 9, 179-181 (2012).

24. Marchini, J., Howie, B., Myers, S., McVean, G. \& Donnelly, P. A new multipoint method for genome-wide association studies by imputation of genotypes. Nat. Genet. 39, 906-913 (2007).

25. Cordell, H. J. et al. Genome-wide association study of multiple congenital heart disease phenotypes identifies a susceptibility locus for atrial septal defect at chromosome 4p16. Nat. Genet. 45, 822-824 (2013).

26. Yang, T. P. et al. Genevar: a database and Java application for the analysis and visualization of SNP-gene associations in eQTL studies. Bioinformatics 26, 2474-2476 (2010).

27. Grundberg, E. et al. Global analysis of DNA methylation variation in adipose tissue from twins reveals links to disease-associated variants in distal regulatory elements. Am. J. Hum. Genet. 93, 876-890 (2013).

28. Xia, K. et al. seeQTL: a searchable database for human eQTLs. Bioinformatics 28, 451-452 (2012).

29. Maurano, M. T. et al. Systematic localization of common disease-associated variation in regulatory DNA. Science 337, 1190-1195 (2012).

30. Thurman, R. E. et al. The accessible chromatin landscape of the human genome. Nature 489, 75-82 (2012).

31. Hoffman, M. M. et al. Integrative annotation of chromatin elements from ENCODE data. Nucleic Acids Res. 41, 827-841 (2013).

\section{Acknowledgements}

This study was funded by the Isaac Newton Trust, PBC Foundation, Medical Research Council (grant reference MR/L001489/1), Wellcome Trust (grants 085925), Ontario Physician Services Inc., Canadian Institutes for Health Research (MOP74621), the Ontario Research Fund (RE01-061) and National Institutes of Health (R01DK091823 and RO1DK80670). H.J.C. is a Wellcome Trust Research Fellow in the Basic Biomedical Science (087436 and 102858). C.S.G. is a Moore Investigator in Data-Driven Discovery (GBMF4552) and was supported in part by GM103534. G.F.M. is a post-doctoral clinical fellow of the National Institute for Health Research Rare Diseases (NIHR-RD) initiative. R.N.S. and G.M.H. receive salary support from a MRC-stratified medicine award (UK-PBC). C.I.A. is partially supported by P30 CA023108. K.A.S. is supported by the Sherman Family Chair in Genomic Medicine and a Canada Research Chair award. This study makes use of data generated by the WTCCC 2 and WTCCC 3 , funded by the Wellcome Trust under awards 085475 and 090355 . Access to genotype data from the
TwinsUK cohort was kindly provided by the Department of Twin Research and Genetic Epidemiology at King's College London. TwinsUK is funded by the Wellcome Trust and the European Community's Seventh Framework Programme (FP7/2007-2013) and also receives support from the UK Department of Health via a National Institute for Health Research (NIHR) Comprehensive Biomedical Research Centre award to Guy's \& St Thomas' NHS Foundation Trust in partnership with King's College London. TwinsUK SNP genotyping was performed by the Wellcome Trust Sanger Institute and the National Eye Institute via the US National Institutes of Health/Center for Integrated Disease Research. Data derived from CGEMs studies (http://dceg.cancer.gov/research/how-westudy/genomic-studies/cgems-summary) were retrieved from dbGAP under a protocol led by Dr Amos. Data derived from the New York Cancer Project were provided by Dr Peter Gregersen.

\section{Author contributions}

This study was initially conceived and designed by H.J.C., G.F.M., C.A.A., M.F.S., R.N.S C.I.A. and K.A.S.; the collection and processing of samples for the study were supervised and coordinated by G.F.M., G.M.H., D.P., A.L., D.C., M.E.G., P.I., K.N.L., M.F.S., R.N.S and K.A.S.; lab work was supervised by G.X. and the statistical analyses of the data were performed by H.J.C., G.F.M., G.M.H., C.S.G., C.I.A. and K.A.S.; the paper was written primarily by H.J.C., G.F.M., G.M.H., C.S.G., C.I.A. and K.A.S. and critically reviewed and revised by all of the above authors.

\section{Additional information}

Supplementary Information accompanies this paper at http://www.nature.com/ naturecommunications

\section{Competing financial interests: The authors declare no competing financial interests}

Reprints and permission information is available online at http://npg.nature.com/ reprintsandpermissions/

How to cite this article: Cordell, H. J. et al. International genome-wide meta-analysis identifies new primary biliary cirrhosis risk loci and targetable pathogenic pathways. Nat Commun. 6:8019 doi: 10.1038/ncomms9019 (2015).

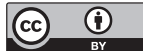

This work is licensed under a Creative Commons Attribution 4.0 International License. The images or other third party material in this article are included in the article's Creative Commons license, unless indicated otherwise in the credit line; if the material is not included under the Creative Commons license, users will need to obtain permission from the license holder to reproduce the material. To view a copy of this license, visit http://creativecommons.org/licenses/by/4.0/

\section{Canadian-US PBC Consortium}

Erik M. Schlicht ${ }^{7}$, Craig Lammert ${ }^{7}$, Elizabeth J. Atkinson ${ }^{19}$, Landon L. Chan ${ }^{19}$, Mariza de Andrade ${ }^{19}$, Tobias Balschun ${ }^{20}$, Andrew L. Mason 21, Robert P. Myers ${ }^{22}$, Jinyi Zhang ${ }^{23}$, Piotr Milkiewicz ${ }^{24}$, Jia Qu ${ }^{25}$, Joseph A. Odin ${ }^{26}$, Velimir A. Luketic ${ }^{27}$, Bruce R. Bacon ${ }^{28}$, Henry C. Bodenheimer Jr29, Valentina Liakina ${ }^{30}$, Catherine Vincent ${ }^{31}$, Cynthia Levy ${ }^{32} \&$ Peter K. Gregersen ${ }^{33}$

${ }^{19}$ Division of Biomedical Statistics and Informatics, Mayo Clinic, Rochester, MN 55905, USA. ${ }^{20}$ Institute of Clinical Molecular Biology, Christian-Albrechts-University of Kiel, 24118 Kiel, Germany. ${ }^{21}$ Department of Medicine, University of Alberta, Edmonton, Alberta T6G 2G3, Canada. ${ }^{22}$ Liver Unit, Division of Gastroenterology and Hepatology, University of Calgary, Calgary, Alberta T6G 2X8, Canada. ${ }^{23}$ Department of Medicine, Mount Sinai Hospital, Lunenfeld-Tanenbaum Research Institute, Toronto, Ontario M5G 1X5, Canada. ${ }^{24}$ Liver Unit, Pomeranian Medical School, $71-252$ Szczecin, Poland. ${ }^{25}$ Department of Optometry and Ophthalmology, Wenzhou Medical University, Wenzhou, Zhejiang 325035, China. ${ }^{26}$ Division of Liver Diseases, Mount Sinai School of Medicine, New York, New York 10029, USA. ${ }^{27}$ Department of Gastroenterology, Virginia Commonwealth University, Richmond, Virginia 23298-0341, USA. ${ }^{28}$ Division of Gastroenterology and Hepatology, Saint Louis University School of Medicine, St Louis, Missouri 63110-0250, USA. ${ }^{29}$ Department of Medicine, Beth Israel Medical Center, Albert Einstein College of Medicine, New York, New York 10461, USA. ${ }^{30}$ Centre of Hepatology, Gastroenterology and Dietetics, Vilnius University, Vilnius 01513, Lithuania. ${ }^{31}$ Universite de Montreal Hospital Centre, Saint-Luc Hospital, Montreal, Quebec H2X 3J4, Canada. ${ }^{32}$ Center for Liver Diseases Division of Hepatology, University of Miami School of Medicine, Miami, Florida 33136, USA.

${ }^{33}$ Feinstein Institute for Medical Research, North Shore LIJ Health System, Manhasset, New York 11030, USA.

\section{Italian PBC Genetics Study Group}

Piero L. Almasio ${ }^{34}$, Domenico Alvaro ${ }^{35}$, Pietro Andreone ${ }^{36}$, Angelo Andriulli ${ }^{37}$, Cristina Barlassina ${ }^{38}$, Pier Maria Battezzati ${ }^{39}$, Antonio Benedetti ${ }^{40}$, Francesca Bernuzzi ${ }^{41}$, llaria Bianchi ${ }^{41}$, Maria Consiglia Bragazzi ${ }^{42}$, Maurizia Brunetto ${ }^{43}$, Savino Bruno ${ }^{41}$, Giovanni Casella ${ }^{44}$, Barbara Coco ${ }^{43}$, Agostino Colli ${ }^{45}$, Massimo Colombo ${ }^{46}$, Silvia Colombo ${ }^{47}$, Carmela Cursaro ${ }^{36}$, Lory Saveria Crocè ${ }^{48}$, Andrea Crosignani ${ }^{39}$, 
Maria Francesca Donato ${ }^{46}$, Gianfranco Elia ${ }^{49}$, Luca Fabris ${ }^{50}$, Carlo Ferrari ${ }^{49}$, Annarosa Floreani ${ }^{51}$, Barbara Foglieni ${ }^{11}$, Rosanna Fontana ${ }^{37}$, Andrea Galli52, Roberta Lazzari ${ }^{51}$, Fabio Macaluso ${ }^{34}$, Federica Malinverno ${ }^{46}$, Fabio Marra ${ }^{53}$, Marco Marzioni ${ }^{40}$, Alberto Mattalia ${ }^{54}$, Renzo Montanari ${ }^{55}$, Lorenzo Morini ${ }^{56}$, Filomena Morisco 57 , Mousa Hani S. ${ }^{41}$, Luigi Muratori ${ }^{36}$, Paolo Muratori ${ }^{36}$, Grazia A. Niro ${ }^{37}$, Vincenzo O. Palmieri ${ }^{58}$, Antonio Picciotto ${ }^{59}$, Mauro Podda 41 , Piero Portincasa ${ }^{58}$, Vincenzo Ronca ${ }^{41}$, Floriano Rosina 60 , Sonia Rossi ${ }^{41}$, Ilaria Sogno ${ }^{41}$, Giancarlo Spinzi ${ }^{61}$, Marta Spreafico ${ }^{11}$, Mario Strazzabosco ${ }^{62,63}$, Sonia Tarallo60, Mirko Tarocchi ${ }^{52}$, Claudio Tiribelli ${ }^{48}$, Pierluigi Toniutto ${ }^{64}$, Maria Vinci ${ }^{65}$ \& Massimo Zuin ${ }^{39}$

\footnotetext{
${ }^{34}$ Gastroenterology \& Hepatology Unit, Di.Bi.M.I.S., University of Palermo, Piazza delle Cliniche 2, 90127 Palermo, Italy. ${ }^{35}$ Eleonora Lorillard Spencer-Cenci Foundation, 00100, Rome, Italy. ${ }^{36}$ Dipartimento di Scienze Mediche e Chirurgiche, Università di Bologna e Dipartimento dell'Apparato Digerente, Azienda Ospedaliero-Universitaria di Bologna, Policlinico Sant'Orsola Malpighi, via Massarenti 9, 40138 Bologna, Italy. ${ }^{37}$ Division of Gastroenterology, IRCCS Casa Sollievo della Sofferenza Hospital, viale Cappuccini 1, San Giovanni Rotondo, Italy, 71013. ${ }^{38}$ Department of Health Sciences, Università degli Studi di Milano, via di Rudinì, 20142 Milan, Italy. ${ }^{39}$ Liver Unit, Department of Health Sciences, San Paolo Hospital Medical School, University of Milan, via di Rudinì, 20142 Milan, Italy. ${ }^{40}$ Department of Gastroenterology, Università Politecnica delle Marche, Ospedali Riuniti University Hospital, via Tronto 10, 60126 Ancona, Italy. ${ }^{41}$ Center for Autoimmune Liver Diseases, Liver Unit, Department of Medicine, Humanitas Clinical and Research Center, via Manzoni 56, Rozzano, 20089 Milan, Italy. ${ }^{42}$ Department of Medico-Surgical Sciences and Biotechnologies, Polo Pontino, Sapienza University of Rome, Viale Università 37,00185 Rome, Italy. ${ }^{43}$ Hepatology Unit, Azienda Ospedaliera Universitaria Pisana, via Roma 67, 56126 Pisa, Italy. ${ }^{44}$ Gastroenterology and Liver Unit, Medical Department, Desio Hospital, via Mazzini, 5, 20033 Desio (MB), Italy. ${ }^{45}$ Department of Internal Medicine, AO Provincia di Lecco, via dell'Eremo 9/11, 23900 Lecco, Italy. ${ }^{46}$ Division of Gastroenterology, Fondazione IRCCS Ca' Granda, Ospedale Maggiore Policlinico, Via Francesco Sforza 33, 20122 Milan, Italy. ${ }^{47}$ Liver Unit, Treviglio Hospital, P.le Ospedale 1, 24047 Treviglio (BG), Italy. ${ }^{48}$ Clinica Patologie del Fegato, University of Trieste, \& Fondazione Italiana Fegato (FIF), Cattinara Hospital, 34149 Trieste, Italy. ${ }^{49}$ Unit of Infectious Diseases and Hepatology, Azienda Ospedaliero-Universitaria di Parma, via Gramsci 14,43126 Parma, Italy. ${ }^{50}$ Department of Molecular Medicine, University of Padova School of Medicine, Padova, Via Gabelli, 63, 35121 Padova Italy. ${ }^{51}$ Department of Surgery, Oncology and Gastroenterology, University of Padova, via Giustiniani 2, 35128 Padua, Italy. ${ }^{52}$ Gastroenterology Unit, University of Florence, viale Pieraccini 6, 50139 Florence, Italy. ${ }^{53}$ Department of Experimental and Clinical Medicine, University of Florence, Largo Brambilla 3, 50134 Florence, Italy.

${ }^{54}$ SC Gastroenterologia Endoscopia Digestiva, Santa Croce Carle Hospital, via Coppino 26, 12100 Cuneo, Italy. ${ }^{55}$ Division of Gastroenterology, Sacro Cuore Don Calabria Hospital, Negrar, 37024 Verona, Italy. ${ }^{56}$ Division of Internal Medicine, Magenta Hospital, Via Al Donatore di Sangue 50, 20013 Magenta, Italy. ${ }^{57}$ Department of Clinical Medicine and Surgery, Gastroenterology Unit, University of Naples Federico II 80138, Italy. ${ }^{58}$ Department of Biomedical Sciences and Human Oncology, University Medical School, Bari, Piazza Giulio Cesare 11, Bari 70124, Italy. ${ }^{59}$ Department of Internal Medicine, University of Genoa, viale Benedetto XV 6, 16145 Genoa, Italy. ${ }^{60}$ Division of Gastroenterology \& Hepatology, Center for Predictive Medicine, Gradenigo Hospital, C.so Regina Margherita 10, 10153 Turin, Italy. ${ }^{61}$ Division of Gastroenterology, Valduce Hospital, via Dante 11, 22100 Como, Italy. ${ }^{62}$ Liver Center, Yale University School of Medicine, New Haven, Connecticut 06511, USA. ${ }^{63}$ Section of Gastroenterology, Department of Translational Medicine and Surgery, University of Milan-Bicocca, via Pergolesi 33, 20900 Monza, Italy. ${ }^{64}$ Department of Internal Medicine, Liver Transplant Unit, University of Udine, P.zale S.M. Misericordia 1, 33100 Udine, Italy. ${ }^{65}$ Division of Hepatology, Niguarda Ca' Granda Hospital, Piazza Ospedale Maggiore 3, 20162 Milan, Italy.
}

\section{UK-PBC Consortium}

Chin Lye Ch'ng 66,67, Mesbah Rahman ${ }^{68}$, Tom Yapp ${ }^{69}$, Richard Sturgess ${ }^{70}$, Christopher Healey ${ }^{71}$, Marek Czajkowski ${ }^{72,73,74}$, Anton Gunasekera ${ }^{75,76}$, Pranab Gyawali ${ }^{77}$, Purushothaman Premchand ${ }^{78}$, Kapil Kapur ${ }^{79}$, Richard Marley ${ }^{80}$, Graham Foster ${ }^{80}$, Alan Watson ${ }^{81}$, Aruna Dias ${ }^{82}$, Javaid Subhani ${ }^{83}$, Rory Harvey ${ }^{84}$, Roger McCorry ${ }^{85}$, David Ramanaden ${ }^{86}$, Jaber Gasem ${ }^{87}$, Richard Evans ${ }^{88}$,

Thiriloganathan Mathialahan ${ }^{89}$, Christopher Shorrock ${ }^{90}$, George Lipscomb ${ }^{91}$, Paul Southern ${ }^{92}$, Jeremy Tibble ${ }^{93,94}$, David Gorard95,96,97, Altaf Palegwala ${ }^{98}$, Susan Jones 99,100 , Marco Carbone ${ }^{101}$, Mohamed Dawwas ${ }^{101}$, Graeme Alexander ${ }^{101}$, Sunil Dolwani ${ }^{102,103}$, Martin Prince ${ }^{104}$, Matthew Foxton ${ }^{105}$, David Elphick ${ }^{106}$, Harriet Mitchisonn 107 , lan Gooding ${ }^{108}$, Mazn Karmo ${ }^{109}$, Sushma Saksena ${ }^{110,111,}$ Mike Mendall122,113, Minesh Patel114,115,116, Roland Ede ${ }^{117,118}$, Andrew Austin ${ }^{119}$, Joanna Sayer ${ }^{120,121}$, Lorraine Hankey ${ }^{122}$, Christopher Hovell122, Neil Fisher ${ }^{123}$, Martyn Carter ${ }^{124,125}$, Konrad Koss ${ }^{126}$, Andrzej Piotrowicz ${ }^{127,128,129,130}$, Charles Grimley 131,132, David Neal133,134, Guan Lim³5, Sass Levi136,137, Aftab Ala ${ }^{138}$, Andrea Broad ${ }^{139}$, Athar Saeed ${ }^{139}$, Gordon Wood ${ }^{140}$, Jonathan Brown ${ }^{141,142}$, Mark Wilkinson ${ }^{143,144}$, Harriet Gordon ${ }^{145}$, John Ramage ${ }^{146}$, Jo Ridpath ${ }^{147}$, Theodore Ngatchu148,149,150, Bob Grover ${ }^{151}$, Syed Shaukat ${ }^{152}$, Ray Shidrawi ${ }^{153}$, George Abouda ${ }^{154,155}$, Faiz Ali156, lan Rees ${ }^{157}$, Imroz Salam58, Mark Narain ${ }^{159}$, Ashley Brown 160,161,162, Simon Taylor-Robinson ${ }^{162}$, Simon Williams ${ }^{163}$, Leonie Grellier ${ }^{164}$, Paul Banim ${ }^{165}$, Debasish Das 166, Andrew Chilton 166, Michael Heneghan 167, Howard Curtis ${ }^{168,169}$, Markus Gess ${ }^{170}$, lan Drake ${ }^{171,172}$, Mark Aldersley 173,174, Mervyn Davies ${ }^{173,174}$, Rebecca Jones ${ }^{173,174}$, Alastair McNair ${ }^{175}$, Raj Srirajaskanthan ${ }^{176}$, Maxton Pitcher ${ }^{177,178}$, Sambit Sen ${ }^{179}$, George Bird ${ }^{180,181}$, Adrian Barnardo ${ }^{182}$, 
Paul Kitchen ${ }^{182}$, Kevin Yoong ${ }^{183}$, Oza Chirag ${ }^{184,185}$, Nurani Sivaramakrishnan ${ }^{186}$, George MacFaul ${ }^{187}$, David Jones ${ }^{188}$, Amir Shah ${ }^{189}$, Chris Evans ${ }^{190}$, Subrata Saha ${ }^{191}$, Katharine Pollock ${ }^{192,193}$, Peter Bramley ${ }^{194,195}$, Ashis Mukhopadhya196,197,198, Andrew Fraser196,197,198, Peter Mills 199,200,201,202,203,204,

Christopher Shallcross 205,206, Stewart Campbell207,208,209, Andrew Bathgate210,211,212, Alan Shepherd213, John Dillon ${ }^{214}$, Simon Rushbrook ${ }^{215}$, Robert Przemioslo ${ }^{216}$ Christopher Macdonald 217,218, Jane Metcalf 219,220 , Udi Shmueli221, Andrew Davis 222,223, Asifabbas Naqvi224,225,226, Tom Lee 227,228, Stephen D Ryder 229,230, Jane Collier ${ }^{231}$, Howard Klass 232,233,234,235, Mary Ninkovic ${ }^{236,237}$, Matthew Cramp ${ }^{238}$, Nicholas Sharer ${ }^{239}$, Richard Aspinall240, Patrick Goggin240, Deb Ghosh241,242, Andrew Douds ${ }^{243}$, Barbara Hoeroldt244, Jonathan Booth 245 , Earl Williams 246 , Hyder Hussaini 247 , William Stableforth ${ }^{247}$, Reuben Ayres ${ }^{248}$, Douglas Thorburn ${ }^{249}$, Eileen Marshall249, Andrew Burroughs ${ }^{249}$, Steven Mann250,251, Martin Lombard ${ }^{252}$, Paul Richardson 252, Imran Patanwala 252, Julia Maltby ${ }^{253}$, Matthew Brookes ${ }^{254}$, Ray Mathew 255,256, Samir Vyas 257, Saket Singhal258, Dermot Gleeson259,260, Sharat Misra 261,262, Jeff Butterworth ${ }^{263,264}$, Keith George 265, Tim Harding266,267, Andrew Douglass 268,269, Simon Panter 270, Jeremy Shearman ${ }^{271}$, Gary Bray 272, Graham Butcher273,274, Daniel Forton275, John Mclindon276,277, Matthew Cowan ${ }^{279}$, Gregory Whatley 280, Aditya Mandal281,282, Hemant Gupta ${ }^{281,282}$, Pradeep Sanghi281,282, Sanjiv Jain ${ }^{283}$, Steve Pereira ${ }^{284}$, Geeta Prasad ${ }^{285}$, Gill Watts ${ }^{285}$, Mark Wright ${ }^{286}$, James Neuberger ${ }^{287}$, Fiona Gordon ${ }^{288}$, Esther Unitt 289 , Allister Grant 290,291,292, Toby Delahooke290,291,292, Andrew Higham293, Alison Brind 294 , Mark Cox ${ }^{295}$, Subramaniam Ramakrishnan ${ }^{296}$, Alistair King 297,298,299, Carole Collins ${ }^{300, ~ S i m o n ~ W h a l l e y ~}{ }^{301,302,}$ Andy Li303, Jocelyn Fraser ${ }^{304}$, Andrew Bell ${ }^{305}$, Voi Shim Wong 306 , Amit Singhal ${ }^{307,308}$, lan Gee 309,310,311, Yeng Ang 312 , Rupert Ransford 313 , James Gotto ${ }^{314}$, Charles Millson 315,316,317, Jane Bowles ${ }^{318}$, Caradog Thomas 66,67,68,69,157,158, Melanie Harrison ${ }^{70}$, Roman Galaska 71 , Jennie Kendall ${ }^{72,73,74,}$ Jessica Whiteman $72,73,74$, Caroline Lawlor ${ }^{75,76}$, Catherine Gray ${ }^{75,76}$, Keith Elliott ${ }^{79}$, Caroline Mulvaney-Jones $86,87,88,89$, Lucie Hobson $86,87,88,89$, Greta Van Duyvenvoorde ${ }^{90}$, Alison Loftus ${ }^{91}$, Katie Seward ${ }^{92}$, Ruth Penn $95,96,97$, Jane Maiden ${ }^{98}$, Rose Damant ${ }^{98}$, Janeane Hails ${ }^{101}$, Rebecca Cloudsdale ${ }^{102,103}$, Valeria Silvestre ${ }^{105}$, Sue Glenn ${ }^{106}$, Eleanor Dungca ${ }^{107}$, Natalie Wheatley ${ }^{108}$, Helen Doyle ${ }^{109}$, Melanie Kent ${ }^{110,111}$, Caroline Hamilton 114,115,116, Delyth Braim 114,115,116, Helen Wooldridge ${ }^{117}$, Rachel Abrahams ${ }^{117}$, Alison Paton ${ }^{119}$, Nicola Lancaster 120,121, Andrew Gibbins ${ }^{122}$, Karen Hogben ${ }^{122}$, Phillipa Desousa ${ }^{124,125}$, Florin Muscariu ${ }^{129,130,}$ Janine Musselwhite ${ }^{129,130}$, Alexandra McKay ${ }^{132}$, LaiTing Tan ${ }^{135}$, Carole Foale ${ }^{138}$, Jacqueline Brighton ${ }^{138}$, Kerry Flahive ${ }^{140}$, Estelle Nambela ${ }^{141,142}$, Paula Townshend ${ }^{141,142}$, Chris Ford ${ }^{141,142}$, Sophie Holder ${ }^{141,142}$, Caroline Palmer ${ }^{145,146}$, James Featherstone ${ }^{147}$, Mariam Nasseri ${ }^{151}$, Joy Sadeghian ${ }^{153}$, Bronwen Williams ${ }^{154,155}$, Carol Thomas ${ }^{156}$, Sally-Ann Rolls ${ }^{156}$, Abigail Hynes ${ }^{159}$, Claire Duggan ${ }^{159}$, Sarah Jones ${ }^{159}$, Mary Crossey ${ }^{160,161,162}$, Glynis Stansfield ${ }^{163}$, Carolyn MacNicol ${ }^{163}$, Joy Wilkins ${ }^{164}$, Elva Wilhelmsen ${ }^{165}$, Parizade Raymode ${ }^{166}$, Hye-Jeong Lee ${ }^{167}$, Emma Durant ${ }^{171,172}$, Rebecca Bishop ${ }^{173,174}$, Noma Ncube ${ }^{175,176}$, Sherill Tripoli ${ }^{177,178}$, Rebecca Casey ${ }^{180,181}$, Caroline Cowley ${ }^{182}$, Richard Miller ${ }^{183}$, Kathryn Houghton ${ }^{188}$, Samantha Ducker ${ }^{188}$, Fiona Wright ${ }^{189}$, Bridget Bird ${ }^{191}$, Gwen Baxter ${ }^{191}$, Janie Keggans ${ }^{191}$, Maggie Hughes ${ }^{194,195}$, Emma Grieve ${ }^{196}$, Karin Young ${ }^{196}$, D. Williams ${ }^{197}$, Kate Ocker ${ }^{199}$, Frances Hines 205,206, Kirsty Martin210,212, Caron Innes 213,214 , Talal Valliani ${ }^{216}$, Helen Fairlamb 217,218, Sarah Thornthwaite 217,218, Anne Eastick 219,220, Elizabeth Tanqueray 221, Jennifer Morrison 222 , Becky Holbrook ${ }^{222}$, Julie Browning 224,226, Kirsten Walker227,228, Susan Congreave 229,230, Juliette Verheyden 229,230 , Susan Slininger 229,230 , Lizzie Stafford ${ }^{231}$, Denise O'Donnell ${ }^{231}$, Mark Ainsworth ${ }^{231}$, Susan Lord ${ }^{118}$, Linda Kent232,233,234,235, Linda March238, Christine Dickson ${ }^{239}$, Diane Simpson ${ }^{239}$, Beverley Longhurst ${ }^{240}$, Maria Hayes ${ }^{240}$, Ervin Shpuza241,242, Nikki White241,242, Sarah Besley244, Sallyanne Pearson ${ }^{244}$, Alice Wright ${ }^{245}$, Linda Jones ${ }^{245}$, Emma Gunter ${ }^{246}$, Hannah Dewhurst ${ }^{246}$, Anna Fouracres 247 , Liz Farrington 247, Lyn Graves ${ }^{247}$, Suzie Marriott ${ }^{248}$, Marina Leoni ${ }^{249}$, David Tyrer ${ }^{252}$, 
Kate Martin 252, Lola Dali-kemmery ${ }^{253}$, Victoria Lambourne ${ }^{253}$, Marie Green254, Dawn Sirdefield 255,256, Kelly Amor ${ }^{255}$, Julie Colley ${ }^{258}$, Bal Shinder 258 , Jayne Jones ${ }^{260}$, Marisa Mills ${ }^{260}$, Mandy Carnahan263,264, Natalie Taylor 265 , Kerenza Boulton ${ }^{265}$, Julie Tregonning 268,269, Carly Brown 270 , Gayle Clifford 270 , Emily Archer 271 , Maria Hamilton 273,274, Janette Curtis 278 , Tracey Shewan ${ }^{279}$, Sue Walsh280, Karen Warner 281,282 , Kimberley Netherton 283, Mcdonald Mupudzi ${ }^{286}$, Bridget Gunson287, Jane Gitahi288, Denise Gocher 289 , Sally Batham 290,291, Hilary Pateman290,291, Senayon Desmennu290,291, Jill Conder ${ }^{293}$, Darren Clement ${ }^{294}$, Susan Gallagher ${ }^{294}$, Jacky Orpe 256 , PuiChing Chan ${ }^{296}$, Lynn Currie 297,298,299, Lynn O’Donohoe 297,298,299, Metod Oblak ${ }^{300, ~ L i s a ~ M o r g a n ² 02, ~ M a r i e ~ Q u i n n ³ 3, ~ I s o b e l ~ A m e y ~}{ }^{304}$, Yolanda Baird ${ }^{304}$, Donna Cotterill ${ }^{305}$, Lourdes Cumlat ${ }^{306}$, Louise Winter ${ }^{312}$, Sandra Greer ${ }^{312}$, Katie Spurdle ${ }^{314}$, Joanna Allison ${ }^{314}$, Simon Dyer ${ }^{315,316}$, Helen Sweeting ${ }^{317}$ \& Jean Kordula ${ }^{318}$

${ }^{66}$ Abertawe Bro Morgannwg University Health Board, Morriston Hospital, Heol Maes Eglwys, Morriston, Swansea SA6 6NL, UK. ${ }^{67}$ Abertawe Bro Morgannwg University Health Board, Singleton Hospital, Sketty Lane, Sketty, Swansea SA2 8QA, UK. ${ }^{68}$ Abertawe Bro Morgannwg University Health Board, Neath Port Talbot Hospital, Baglan Way, Port Talbot SA12 7BX, UK. ${ }^{69}$ Abertawe Bro Morgannwg University Health Board, Princess of Wales Hospital, Coity Road, Bridgend CF31 1RQ, UK. ${ }^{70}$ Aintree University Hospitals NHS Foundation Trust, Aintree University Hospital, Longmoor Lane, Liverpool L9 $7 A L$, UK. ${ }^{71}$ Airedale NHS Foundation Trust, Airedale General Hospital, Skipton Road, Steeton, Keighley BD20 6TD, UK. ${ }^{72}$ Aneurin Bevan University Health Board, Nevill Hall Hospital, Brecon Road, Abergavenny NP7 7EG, UK. ${ }^{73}$ Aneurin Bevan University Health Board, Royal Gwent Hospital, Cardiff Road, Newport NP20 2 UB, UK. ${ }^{74}$ Aneurin Bevan University Health Board, Ysbyty Ystrad Fawr, Ystrad Fawr Way, Ystrad Mynach, Hengoed CF82 7EP, UK. ${ }^{75}$ Ashford \& St Peter's Hospitals NHS Foundation Trust, Ashford Hospital, London Road, Ashford TW15 3AA, UK. ${ }^{76}$ Ashford \& St Peter's Hospitals NHS Foundation Trust, St Peter's Hospital, Guildford Road, Chertsey KT16 OPZ, UK. ${ }^{77}$ Barking, Havering and Redbridge University Hospitals NHS Trust, King George Hospital, Barley Lane, Ilford IG3 8YB, UK. ${ }^{78}$ Barking, Havering and Redbridge University Hospitals NHS Trust, Queen's Hospital, Rom Valley Way, Romford RM7 OAG, UK. ${ }^{79}$ Barnsley Hospital NHS Foundation Trust, Barnsley Hospital, Gawber Road, Barnsley S75 2EP, UK. ${ }^{80}$ Barts Health NHS Trust, The Royal London Hospital, Whitechapel Road, Whitechapel, London E1 1BB, UK. ${ }^{81}$ Barts Health NHS Trust, Whipps Cross University Hospital, Whipps Cross Road, Leytonstone, London E11 1NR, UK. ${ }^{82}$ Barts Health NHS Trust, Newham University Hospital, Glen Road, Plaistow, London E13 8SL, UK. ${ }^{83}$ Basildon and Thurrock University Hospitals NHS Foundation Trust, Basildon University Hospital, Nethermayne, Basildon SS16 5NL, UK. ${ }^{84}$ Bedford Hospital NHS Trust, Bedford Hospital, Kempston Road, Bedford MK42 9DJ, UK. ${ }^{85}$ Belfast Health and Social Care Trust, Royal Victoria Hospital, 274 Grosvenor Road, Belfast BT12 6BA, UK. ${ }^{86}$ Betsi Cadwaladr University Health Board, Glan Clwyd Hospital, Rhyl LL18 5UJ, UK. ${ }^{87}$ Betsi Cadwaladr University Health Board, Ysbyty Gwynedd, Penrhosgarnedd, Bangor LL57 2PW, UK. ${ }^{88}$ Betsi Cadwaladr University Health Board, Llandudno General Hospital, Hospital Road, Llandudno LL30 1LB, UK. ${ }^{89}$ Betsi Cadwaladr University Health Board, Wrexham Maelor Hospital, Croesnewydd Road, Wrexham LL13 7TD, UK. ${ }^{90}$ Blackpool Teaching Hospitals NHS Foundation Trusts, Blackpool Victoria Hospital, Whinney Heys Road, Blackpool FY3 8NR, UK. ${ }^{91}$ Bolton NHS Foundation Trust, Royal Bolton Hospital, Minerva Road, Farnworth, Bolton BL4 OJR, UK. ${ }^{92}$ Bradford Teaching Hospitals NHS Foundation Trust, Bradford Royal Infirmary, Duckworth Lane, Bradford BD9 6RJ, UK. ${ }^{33}$ Brighton and Sussex University Hospitals NHS Trust, Princess Royal Hospital, Lewes Road, Haywards Heath RH16 4EX, UK. ${ }^{4}$ Brighton and Sussex University Hospitals NHS Trust, Royal Sussex County Hospital, Eastern Road, Brighton BN2 5BE, UK. ${ }^{95}$ Buckinghamshire Healthcare NHS Trust, Amersham Hospital, Whielden Street, Amersham HP7 OJD, UK. ${ }^{96}$ Buckinghamshire Healthcare NHS Trust, Stoke Mandeville Hospital, Mandeville Road, Aylesbury HP21 8 AL, UK.

${ }^{97}$ Buckinghamshire Healthcare NHS Trust, Wycombe Hospital, Queen Alexandra Road, High Wycombe HP11 2TT, UK. ${ }^{88}$ Burton Hospitals NHS Foundation Trust, Queen's Hospital, Belvedere Road, Burton upon Trent DE13 ORB, UK. ${ }^{99}$ Calderdale And Huddersfield NHS Foundation Trust, Calderdale Royal Hospital, Salterhebble, Halifax HX3 OPW, UK. ${ }^{100}$ Calderdale And Huddersfield NHS Foundation Trust, Huddersfield Royal Infirmary, Acre Street, Lindley, Huddersfield HD3 3EA, UK. ${ }^{101}$ Cambridge University Hospitals NHS Foundation Trust, Addenbrooke's Hospital, Cambridge Biomedical Campus, Hills Road, Cambridge CB2 OQQ, UK. ${ }^{102}$ Cardiff and Vale University Health Board, University Hospital Llandough, Penlan Road, Llandough, Penarth CF64 2XX, UK. ${ }^{03}$ Cardiff and Vale University Health Board, University Hospital of Wales, Heath Park, Cardiff CF14 4XW, UK. ${ }^{104}$ Central Manchester University Hospitals NHS Foundation Trust, Manchester Royal Infirmary, Oxford Road, Manchester M13 9WL, UK. ${ }^{105}$ Chelsea and Westminster Hospital NHS Foundation Trust, Chelsea and Westminster Hospital, 369 Fulham Road, London SW10 9NH, UK. ${ }^{106}$ Chesterfield Royal Hospital NHS Foundation Trust, Chesterfield Royal Hospital, Calow, Chesterfield S44 5BL, UK. ${ }^{107}$ City Hospitals Sunderland NHS Foundation Trust, Sunderland Royal Hospital, Kayll Road, Sunderland SR4 7TP, UK.

${ }^{108}$ Colchester Hospital University NHS Foundation Trust, Colchester General Hospital, Turner Road, Colchester CO4 5JL, UK. ${ }^{109}$ Countess of Chester Hospital NHS Foundation Trust, Countess of Chester Hospital, Countess of Chester Health Park, Liverpool Road, Chester CH2 1UL, UK. ${ }^{110}$ County Durham and Darlington NHS Foundation Trust, Darlington Memorial Hospital, Hollyhurst Road, Darlington DL3 6HX, UK. ${ }^{111}$ County Durham and Darlington NHS Foundation Trust, University Hospital of North Durham, North Road, Durham DH1 5TW, UK. ${ }^{112}$ Croydon Health Services NHS Trust, Croydon University Hospital, 530 London Road, Croydon CR7 7YE, UK. ${ }^{113}$ Croydon Health Services NHS Trust, Purley War Memorial Hospital, 856 Brighton Road, Purley CR8 2YL, UK. ${ }^{114}$ Cwm Taf University Health Board, Prince Charles Hospital, Gurnos, Merthyr Tydfil CF47 9DT, UK. ${ }^{115}$ Cwm Taf University Health Board, Royal Glamorgan Hospital, Ynysmaerdy, Llantrisant, Pontyclun CF72 8XR, UK. ${ }^{116}$ Cwm Taf University Health Board, Ysbyty Cwm Cynon, New Road, Mountain Ash, Rhondda Cynon Taff CF45 4BZ, UK. ${ }^{117}$ Dartford And Gravesham NHS Trust, Darent Valley Hospital, Darenth Wood Road, Dartford DA2 8DA, UK. ${ }^{118}$ Oxleas NHS Foundation Trust, Queen Mary's Hospital Sidcup, Frognal Avenue, Sidcup DA14 6LT, UK. ${ }^{119}$ Derby Hospitals NHS Foundation Trust, Royal Derby Hospital, Uttoxeter Road, Derby DE22 3NE, UK. ${ }^{120}$ Doncaster and Bassetlaw Hospitals NHS Foundation Trust, Bassetlaw Hospital, Blyth Road, Worksop S81 OBD, UK. ${ }^{121}$ Doncaster and Bassetlaw Hospitals NHS Foundation Trust, Doncaster Royal Infirmary, Armthorpe Road, Doncaster DN2 5LT, UK. ${ }^{122}$ Dorset County Hospitals NHS Foundation Trust, Dorset County Hospital, Williams Avenue, Dorchester DT1 2JY, UK. ${ }^{123}$ Dudley Group of Hospitals NHS Trust, Russells Hall Hospital, Pensnett Road, Dudley DY1 2HQ, UK. ${ }^{124}$ East and North Hertfordshire NHS Trust, Lister Hospital, Coreys Mill Lane, Stevenage SG1 4AB, UK. ${ }^{125}$ East and North Hertfordshire NHS Trust, Queen Elizabeth II Hospital, Howlands, Welwyn Garden City AL7 4HQ, UK. ${ }^{126}$ East Cheshire NHS Trust, Macclesfield District General Hospital, Victoria Road, Macclesfield SK10 3BL, UK. ${ }^{127}$ East Kent Hospitals University NHS Foundation Trust, Buckland Hospital, Coombe Valley Road, Dover CT17 OHD, UK. ${ }^{128}$ East Kent Hospitals University NHS Foundation Trust, Kent and Canterbury Hospital, Ethelbert Road, Canterbury CT1 3NG, UK. ${ }^{129}$ East Kent Hospitals University NHS Foundation Trust, Queen Elizabeth The Queen Mother Hospital, St Peters Road, Margate CT9 4AN, UK. ${ }^{130}$ East Kent Hospitals University NHS Foundation Trust, William Harvey Hospital Ashford, Kennington Road, Willesborough, Ashford TN24 OLZ, UK. ${ }^{131}$ East Lancashire Hospitals NHS Trust, Burnley General Hospital, Casterton Avenue, Burnley BB10 2PQ, UK. ${ }^{132}$ East Lancashire Hospitals NHS Trust, Royal Blackburn Hospital, Haslingden Road, Blackburn BB2 3HH, UK. ${ }^{133}$ East Sussex Healthcare NHS Trust, Conquest Hospital, The Ridge, St Leonards-on-Sea TN37 $7 R D$, UK. 
${ }^{134}$ East Sussex Healthcare NHS Trust, Eastbourne District General Hospital, Kings Drive, Eastbourne BN21 2UD. ${ }^{135}$ Epsom and St Helier University Hospitals NHS Trust, Epsom General Hospital, Epsom Hospital, Dorking Road, Epsom KT18 7EG, UK. ${ }^{136}$ Frimley Health NHS Foundation Trust, Heatherwood Hospital, London Road, Ascot SL5 8AA, UK. ${ }^{137}$ Frimley Health NHS Foundation Trust, Wexham Park Hospital, Wexham, Slough SL2 4HL, UK. ${ }^{138}$ Frimley Health NHS Foundation Trust, Frimley Park Hospital, Portsmouth Road, Frimley GU16 7UJ, UK. ${ }^{139}$ Gateshead Health NHS Foundation Trust, Queen Elizabeth Hospital, Sheriff Hill, Gateshead NE9 6SX, UK. ${ }^{140}$ George Eliot Hospital NHS Trust, George Eliot Hospital, Eliot Way, Nuneaton CV10 7DJ, UK. ${ }^{141}$ Gloucestershire Hospitals NHS Foundation Trust, Cheltenham General Hospital, Sandford Road, Cheltenham GL53 7AN, UK. ${ }^{142}$ Gloucestershire Hospitals NHS Foundation Trust, Gloucestershire Royal Hospital, Great Western Road, Gloucester GL1 3NN, UK. ${ }^{143}$ Guy's and St Thomas' NHS Foundation Trust, Guy's Hospital, Great Maze Pond, London SE1 9RT, UK. ${ }^{144}$ Guy's and St Thomas' NHS Foundation Trust, St Thomas' Hospital, Westminster Bridge Road, London SE1 7EH, UK. ${ }^{145}$ Hampshire Hospitals NHS Foundation Trust, Royal Hampshire County Hospital, Romsey Road, Winchester SO22 5DG, UK. 146 Hampshire Hospitals NHS Foundation Trust, Basingstoke and North Hampshire Hospital, Aldermaston Road, Basingstoke RG24 9NA, UK. ${ }^{147}$ Harrogate and District NHS Foundation Trust, Harrogate District Hospital, Lancaster Park Road, Harrogate HG2 7SX, UK. ${ }^{148}$ Heart of England NHS Foundation Trust, Good Hope Hospital, Rectory Road, Sutton Coldfield, Birmingham B75 7RR, UK. ${ }^{149}$ Heart of England NHS Foundation Trust, Heartlands Hospital, Bordesley Green East, Birmingham B9 5SS, UK. ${ }^{150}$ Heart of England NHS Foundation Trust, Solihull Hospital, Lode Lane, Solihull B91 2JL, UK. ${ }^{151}$ Hillingdon Hospitals NHS Foundation Trust, Hillingdon Hospital, Pield Heath Road, Uxbridge UB8 3NN, UK. ${ }^{152}$ Hinchingbrooke Health Care NHS Trust, Hinchingbrooke Hospital, Hinchingbrooke Park, Huntingdon PE29 6NT, UK. ${ }^{153}$ Homerton University Hospital NHS Foundation Trust, Homerton University Hospital, Homerton Row, London E9 6SR, UK. ${ }^{154}$ Hull And East Yorkshire Hospitals NHS Trust, Castle Hill Hospital, Castle Road, Cottingham HU16 5JQ, UK. ${ }^{155}$ Hull And East Yorkshire Hospitals NHS Trust, Hull Royal Infirmary, Anlaby Road, Hull HU3 2JZ, UK. ${ }^{156}$ Hywel Dda University Health Board, Withybush General Hospital, Fishguard Road, Haverfordwest SA61 2PZ, UK. ${ }^{157}$ Hywel Dda University Health Board, Prince Philip Hospital, Bryngwyn Mawr, Dafen, Llanelli SA14 8QF, UK. ${ }^{158}$ Hywel Dda University Health Board, Glangwili General Hospital, Dolgwilli Road, Carmarthen SA31 2AF, UK. ${ }^{159}$ Hywel Dda University Health Board, Bronglais Hospital, Caradog Road, Aberystwyth SY23 1ER, UK. ${ }^{160}$ Imperial College Healthcare NHS Trust, Charing Cross Hospital, Fulham Palace Road, London W6 8RF, UK. ${ }^{161}$ Imperial College Healthcare NHS Trust, Hammersmith Hospital, Du Cane Road, London W12 OHS, UK. ${ }^{162}$ Imperial College Healthcare NHS Trust, St Mary's Hospital, Praed Street, London W2 1NY, UK. ${ }^{163}$ Ipswich Hospital NHS Trust, Ipswich Hospital, Heath Road, Ipswich IP4 5PD, UK. ${ }^{164}$ Isle of Wight NHS Trust, St Mary's Hospital, Parkhurst Road, Newport PO30 5TG, UK. ${ }^{165}$ James Paget University Hospitals NHS Foundation Trust, James Paget Hospital, Lowestoft Road, Gorleston, Great Yarmouth NR31 6LA, UK. ${ }^{166}$ Kettering General Hospital NHS Foundation Trust, Kettering General Hospital, Rothwell Road, Kettering NN16 8UZ, UK. ${ }^{167}$ Kings College Hospital NHS Foundation Trust, King's College Hospital, Denmark Hill, London SE5 9RS, UK. ${ }^{168}$ King's College Hospital NHS Foundation Trust, Beckenham Beacon, 395 Croydon Road, Beckenham BR3 3QL, UK. ${ }^{169}$ King's College Hospital NHS Foundation Trust, Princess Royal University Hospital, Farnborough Common, Orpington BR6 8ND, UK. ${ }^{170}$ Kingston Hospital NHS Foundation Trust, Kingston Hospital, Galsworthy Road, Kingston upon Thames KT2 7 QB, UK. ${ }^{171}$ Lancashire Teaching Hospitals NHS Foundation Trust, Chorley and South Ribble Hospital, Preston Road, Chorley PR7 1PP, UK. ${ }^{172}$ Lancashire Teaching Hospitals NHS Foundation Trust, Royal Preston Hospital, Sharoe Green Lane North, Preston PR2 9HT, UK. ${ }^{173}$ Leeds Teaching Hospitals NHS Trust, Leeds General Infirmary, Great George Street, Leeds LS1 3EX, UK. ${ }^{174}$ Leeds Teaching Hospitals NHS Trust, St James's University Hospital, Beckett Street, Leeds LS9 7TF, UK. ${ }^{175}$ Lewisham and Greenwich NHS Trust, The Queen Elizabeth, Woolwich, Stadium Road, Greenwich SE18 4QH, UK. ${ }^{176}$ Lewisham and Greenwich NHS Trust, Lewisham Hospital, High Street, Lewisham SE13 6LH, UK. ${ }^{177}$ London North West Healthcare NHS Trust, Central Middlesex Hospital, Acton Lane, Park Royal, London NW10 7NS, UK. ${ }^{178}$ London North West Healthcare NHS Trust, Northwick Park and St Mark's Hospitals, Watford Road, Harrow HA1 3UJ, UK. ${ }^{179}$ Luton and Dunstable University Hospital NHS Foundation Trust, Luton and Dunstable University Hospital, Lewsey Road, Luton LU4 ODZ, UK. ${ }^{180}$ Maidstone and Tunbridge Wells NHS Trust, Maidstone Hospital, Hermitage Lane, Maidstone ME16 9QQ, UK. ${ }^{181}$ Maidstone and Tunbridge Wells NHS Trust, Tunbridge Wells Hospital, Tonbridge Road, Pembury, Tunbridge Wells TN2 4QJ, UK. ${ }^{182}$ Medway NHS Foundation Trust, Medway Maritime Hospital, Windmill Road, Gillingham ME7 5NY, UK. ${ }^{183}$ Mid Cheshire Hospitals NHS Foundation Trust, Leighton Hospital, Middlewich Road, CW1 4QJ, UK. ${ }^{184}$ Mid Essex Hospital Services NHS Trust, Broomfield Hospital, Court Road, Chelmsford CM1 7ET, UK. ${ }^{185}$ Mid Essex Hospital Services NHS Trust, St Peters Hospital, Spital Road, Maldon CM9 6EG, UK. ${ }^{186}$ Mid Yorkshire Hospitals NHS Trust, Dewsbury and District Hospital, Halifax Road, Dewsbury WF13 4HS, UK. ${ }^{187}$ Milton Keynes Hospital NHS Foundation Trust, Milton Keynes Hospital, Standing Way, Milton Keynes MK6 5LD, UK. ${ }^{188}$ Newcastle upon Tyne Hospitals NHS Foundation Trust, Freeman Hospital, Freeman Road, High Heaton, Newcastle upon Tyne NE7 7DN, UK. ${ }^{189}$ NHS Ayrshire \& Arran, University Hospital Crosshouse, Kilmarnock Road, Kilmarnock KA2 OBE, UK. ${ }^{190}$ NHS Borders, Borders General Hospital, Melrose TD6 9BS, UK. ${ }^{191}$ NHS Dumfries \& Galloway, Dumfries and Galloway Royal Infirmary, Bankend Road, Dumfries DG1 4AP, UK. ${ }^{192}$ NHS Fife, Queen Margaret Hospital, Whitefield Road, Dunfermline KY12 OSU, UK. ${ }^{193} \mathrm{NHS}$ Fife, Victoria Hospital, Hayfield Road, Kirkcaldy KY2 5AH, UK. ${ }^{194}$ NHS Forth Valley, Forth Valley Royal Hospital, Stirling Road, Larbert FK5 4WR, UK. ${ }^{195}$ NHS Forth Valley, Stirling Community Hospital, Livilands, Stirling FK8 2 AU, UK. ${ }^{196}$ NHS Grampian, Aberdeen Royal Infirmary, Foresterhill, Aberdeen AB25 2ZN, UK. ${ }^{197}$ NHS Grampian, Dr Gray's Hospital, Elgin IV30 1SN, UK. ${ }^{198}$ NHS Grampian, Woolmanhill Hospital, Skene Street, Aberdeen AB25 1LD, UK. ${ }^{199}$ NHS Greater Glasgow and Clyde, Gartnavel General Hospital, 1053 Great Western Road, Glasgow G12 OYN, UK. ${ }^{200}$ NHS Greater Glasgow and Clyde, Glasgow Royal Infirmary, 84 Castle Street, Glasgow G4 OSF, UK. ${ }^{201}$ NHS Greater Glasgow and Clyde, Inverclyde Royal Hospital, Larkfield Road, Greenock PA16 OXN, UK. ${ }^{202}$ NHS Greater Glasgow and Clyde, Royal Alexandra Hospital, Corsebar Road, Paisley PA2 9PN, UK. ${ }^{203}$ NHS Greater Glasgow and Clyde, Southern General Hospital, 1345 Govan Road, Glasgow G51 4TF, UK. ${ }^{204}$ NHS Greater Glasgow and Clyde, Victoria Infirmary, Langside Road, Glasgow G42 9TY, UK. ${ }^{205}$ NHS Highland, Caithness General Hospital, Bankhead Road, Wick KW1 5NS, UK. ${ }^{206}$ NHS Highland, Raigmore Hospital, Old Perth Road, Inverness IV2 3UJ, UK. ${ }^{207}$ NHS Lanarkshire, Hairmyres Hospital, Eaglesham Road, East Kilbride G75 8RG, UK. ${ }^{208}$ NHS Lanarkshire, Monklands Hospital, Monkscourt Avenue, Airdrie ML6 OJS, UK. ${ }^{209}$ NHS Lanarkshire, Wishaw General Hospital, 50 Netherton Street, Wishaw ML2 ODP, UK. ${ }^{210}$ NHS Lothian, Royal Infirmary of Edinburgh, 51 Little France Crescent, Old Dalkeith Road, Edinburgh EH16 4SA, UK. ${ }^{211}$ NHS Lothian, St John's Hospital, Howden Road West, Howden, Livingston EH54 6PP, UK. ${ }^{212}$ NHS Lothian, Western General Hospital, Crewe Road South, Edinburgh EH4 2XU, UK. ${ }^{213}$ NHS Tayside, Perth Royal Infirmary, Taymount Terrace, Perth PH1 1NX, UK. ${ }^{214}$ NHS Tayside, Ninewells Hospital, Dundee DD1 9SY, UK. ${ }^{215}$ Norfolk and Norwich University Hospitals NHS Foundation Trust, Norfolk and Norwich University Hospital, Colney Lane, Norwich NR4 7UY, UK. ${ }^{216}$ North Bristol NHS Trust, Frenchay Hospital, Frenchay Park Road, Bristol BS16 1LE, UK. ${ }^{217}$ North Cumbria University Hospitals NHS Foundation Trust, Cumberland Infirmary, Newtown Road, Carlisle CA2 7HY, UK. ${ }^{218}$ North Cumbria University Hospitals NHS Foundation Trust, West Cumberland Hospital, Hensingham, Whitehaven CA28 8JG, UK. ${ }^{219}$ North Tees and Hartlepool NHS Foundation Trust, University Hospital of Hartlepool, Holdforth Road, Hartlepool TS24 9AH, UK. 220 North Tees and Hartlepool NHS Foundation Trust, University Hospital of North Tees, Hardwick, Stockton on Tees TS19 8PE, UK. ${ }^{221}$ Northampton General Hospital NHS Trust, Northampton General Hospital, Cliftonville, Northampton NN1 5BD, UK. ${ }^{222}$ Northern Devon Healthcare NHS Trust, North Devon District Hospital, Raleigh Park, Barnstaple EX31 4JB, UK. ${ }^{223}$ Northern Health and Social Care Trust, Whiteabbey Hospital, Doagh Road, Newtownabbey BT37 9RH, UK. ${ }^{224}$ Northern Lincolnshire and Goole NHS Foundation Trust, Diana, Princess of Wales Hospital, Scartho Road, Grimsby DN33 2BA, UK. 225 Northern Lincolnshire and Goole NHS Foundation Trust, Goole and District Hospital, Woodland Avenue, Goole DN14 6RX, UK. ${ }^{226}$ Northern Lincolnshire and Goole NHS Foundation Trust, Scunthorpe General Hospital, Cliff Gardens, Scunthorpe DN15 7BH, UK. ${ }^{227}$ Northumbria Healthcare NHS Foundation Trust, Hexham General Hospital, Corbridge Road, Hexham NE46 1QJ, UK. ${ }^{228}$ Northumbria Healthcare NHS Foundation Trust, North Tyneside Hospital, Rake Lane, North Shields NE29 8NH, UK. ${ }^{229}$ Nottingham University Hospitals NHS Trust, Nottingham City Hospital, Hucknall Road, Nottingham NG5 1PB, UK. ${ }^{230}$ Nottingham University Hospitals NHS Trust, Queen's Medical Centre, Derby Road, Nottingham NG7 2UH, UK. ${ }^{231}$ Oxford University Hospitals NHS Trust, John Radcliffe Hospital, Headley Way, Headington, 
Oxford OX3 9DU, UK. ${ }^{232}$ Pennine Acute Hospitals NHS Trust, Fairfield General Hospital, Rochdale Old Road, Bury BL9 7TD, UK. ${ }^{233}$ Pennine Acute Hospitals NHS Trust, North Manchester General Hospital, Delaunays Road, Crumpsall M8 5RB, UK. ${ }^{234}$ Pennine Acute Hospitals NHS Trust, Rochdale Infirmary, Whitehall Street, Rochdale OL12 ONB, UK. ${ }^{235}$ Pennine Acute Hospitals NHS Trust, The Royal Oldham Hospital, Rochdale Road, Oldham OL1 2JH, UK. 236 Peterborough and Stamford Hospitals NHS Foundation Trust, Peterborough City Hospital, Edith Cavell Campus, Bretton Gate, Peterborough PE3 9 GZ, UK. ${ }^{237}$ Peterborough and Stamford Hospitals NHS Foundation Trust, Stamford \& Rutland Hospital, Ryhall Road, Stamford PE9 1UA, UK. ${ }^{238}$ Plymouth Hospitals NHS Trust, Derriford Hospital, Derriford Road, Plymouth PL6 8DH, UK. ${ }^{239}$ Poole Hospital NHS Foundation Trust, Poole Hospital, Longfleet Road, Poole BH15 2JB, UK. ${ }^{240}$ Portsmouth Hospitals NHS Trust, Queen Alexandra Hospital, Cosham, Portsmouth PO6 3LY, UK. ${ }^{241}$ Princess Alexandra Hospital NHS Trust, St Margaret's Hospital, The Plain, Epping CM16 6TN, UK. ${ }^{242}$ Princess Alexandra Hospital NHS Trust, The Princess Alexandra Hospital, Hamstel Road, Harlow CM20 1QX, UK. ${ }^{243}$ Queen Elizabeth Hospital King's Lynn NHS Foundation Trust, The Queen Elizabeth Hospital King's Lynn, Gayton Road, King's Lynn PE30 4ET, UK. ${ }^{244}$ Rotherham NHS Foundation Trust, Rotherham Hospital, Moorgate Road, Rotherham S60 2UD, UK. ${ }^{245}$ Royal Berkshire NHS Foundation Trust, Royal Berkshire Hospital, Craven Road, Reading RG1 5AN, UK. ${ }^{246}$ Royal Bournemouth and Christchurch Hospitals NHS Foundation Trust, Royal Bournemouth Hospital, Castle Lane East, Bournemouth BH7 7DW, UK. ${ }^{247}$ Royal Cornwall Hospitals NHS Trust, Royal Cornwall Hospital, Treliske, Truro TR1 3LJ, UK. ${ }^{248}$ Royal Devon and Exeter NHS Foundation Trust, Royal Devon and Exeter Hospital, Barrack Road, Exeter EX2 5DW, UK. ${ }^{249}$ Royal Free London NHS Foundation Trust, The Royal Free Hospital, Pond Street, London NW3 2QG, UK. ${ }^{250}$ Royal Free London NHS Foundation Trust, Barnet Hospital, Wellhouse Lane, Barnet EN5 3DJ, UK. ${ }^{251}$ Royal Free London NHS Foundation Trust, Chase Farm Hospital, The Ridgeway, Enfield EN2 8JL, UK. ${ }^{252}$ Royal Liverpool and Broadgreen University Hospitals NHS Trust, Royal Liverpool University Hospital, Prescot Street, Liverpool L7 8XP, UK. ${ }^{253}$ Royal United Hospitals Bath NHS Foundation Trust, Royal United Bath Hospital, Combe Park, Bath BA1 3NG, UK. ${ }^{254}$ Royal Wolverhampton Hospitals NHS Trust, New Cross Hospital, Wolverhampton Road, Wolverhampton WV10 OQP, UK. ${ }^{255}$ Royal Wolverhampton Hospitals NHS Trust, Cannock Chase Hospital, Brunswick Road, Cannock WS11 5XY, UK. ${ }^{256}$ University Hospitals of North Midlands NHS Trust, County Hospital, Weston Road, Stafford ST16 3SA, UK. ${ }^{257}$ Salisbury NHS Foundation Trust, Salisbury District Hospital, Salisbury SP2 8BJ, UK. ${ }^{258}$ Sandwell and West Birmingham Hospitals NHS Trust, Sandwell General Hospital, Lyndon, West Bromwich B71 4HJ, UK. ${ }^{259}$ Sheffield Teaching Hospitals NHS Foundation Trust, Northern General Hospital, Herries Road, Sheffield S5 $7 A U$, UK. 260 Sheffield Teaching Hospitals NHS Foundation Trust, Royal Hallamshire Hospital, Glossop Road, Sheffield S10 2JF, UK. ${ }^{261}$ Sherwood Forest Hospitals NHS Foundation Trust, King's Mill Hospital, Mansfield Road, Sutton in Ashfield NG17 4JL, UK. ${ }^{262}$ Sherwood Forest Hospitals NHS Foundation Trust, Newark Hospital, Boundary Road, Newark NG24 4DE, UK. ${ }^{263}$ Shrewsbury and Telford Hospital NHS Trust, Princess Royal Hospital, Apley Castle, Telford TF1 6TF, UK. ${ }^{264}$ Shrewsbury and Telford Hospital NHS Trust, Royal Shrewsbury Hospital, Mytton Oak Road, Shrewsbury SY3 8XQ, UK. ${ }^{265}$ South Devon Healthcare NHS Foundation Trust, Torbay Hospital, Lowes Bridge, Torquay TQ2 7AA, UK. ${ }^{666}$ South Eastern Health and Social Care Trust, Lagan Valley Hospital, 39 Hillsborough Road, Lisburn BT28 1JP, UK. ${ }^{267}$ South Eastern Health and Social Care Trust, Ulster Hospital, Upper Newtownards Road, Dundonald, Belfast BT16 1RH, UK. ${ }^{268}$ South Tees Hospitals NHS Foundation Trust, The James Cook University Hospital, Marton Road, Middlesbrough TS4 3BW, UK. ${ }^{269}$ South Tees Hospitals NHS Foundation Trust, Friarage Hospital, Northallerton DL6 1JG, UK. ${ }^{270}$ South Tyneside NHS Foundation Trust, South Tyneside District Hospital, Harton Lane, South Shields NE34 OPL, UK. ${ }^{271}$ South Warwickshire NHS Foundation Trust, Warwick Hospital, Lakin Road, Warwick CV34 5BW, UK. ${ }^{272}$ Southend University Hospital NHS Foundation Trust, Southend Hospital, Prittlewell Chase, Westcliff-on-Sea SSO ORY, UK. ${ }^{273}$ Southport \& Ormskirk Hospital NHS Trust, Ormskirk District General Hospital, Wigan Road, Ormskirk L39 2AZ, UK. ${ }^{274}$ Southport \& Ormskirk Hospital NHS Trust, Southport and Formby District General Hospital, Town Lane, Kew, Southport PR8 6PN, UK. ${ }^{275}$ St George's University Hospitals NHS Foundation Trust, St George's Hospital, Blackshaw Road, Tooting, London SW17 OQT, UK. ${ }^{276}$ St Helens and Knowsley Teaching Hospitals NHS Trust, St Helens Hospital, Marshalls Cross Road, St Helens WA9 3DA, UK. ${ }^{277}$ St Helens and Knowsley Teaching Hospitals NHS Trust, Whiston Hospital, Warrington Road, Prescot L35 5DR, UK. 278 Stockport NHS Foundation Trust, Stepping Hill Hospital, Poplar Grove, Hazel Grove, Stockport SK2 7JE, UK. ${ }^{279}$ Surrey and Sussex Healthcare NHS Trust, East Surrey Hospital, Canada Avenue, Redhill RH1 5RH, UK. ${ }^{280}$ Tameside Hospital NHS Foundation Trust, Tameside General Hospital, Fountain Street, Ashton-under-Lyne OL6 9RW, UK. ${ }^{281}$ United Lincolnshire Hospitals NHS Trust, Lincoln County Hospital, Greetwell Road, Lincoln LN2 5QY, UK. ${ }^{282}$ United Lincolnshire Hospitals NHS Trust, Grantham and District Hospital, ${ }^{101}$ Manthorpe Road, Grantham NG31 8DG, UK. ${ }^{283}$ United Lincolnshire Hospitals NHS Trust, Pilgrim Hospital Boston, Sibsey Road, Boston PE21 9QS, UK. ${ }^{284}$ University College London Hospitals NHS Foundation Trust, University College Hospital, 235 Euston Road, London NW1 2BU, UK. ${ }^{285}$ University Hospital of South Manchester NHS Foundation Trust, Wythenshawe Hospital, Southmoor Road, Wythenshawe, Manchester M23 9LT, UK. ${ }^{286}$ University Hospital Southampton NHS Foundation Trust, Southampton General Hospital, Tremona Road, Southampton SO16 6YD, UK. ${ }^{287}$ University Hospitals Birmingham NHS Foundation Trust, Queen Elizabeth Hospital, Mindelsohn Way, Edgbaston, Birmingham B15 2GW, UK. ${ }^{288}$ University Hospitals Bristol NHS Foundation Trust, Bristol Royal Infirmary, Upper Maudlin Street, Bristol BS2 8HW, UK. ${ }^{289}$ University Hospitals Coventry and Warwickshire NHS Trust, University Hospital, Clifford Bridge Road, Coventry CV2 2DX, UK. ${ }^{290}$ University Hospitals of Leicester NHS Trust, Glenfield Hospital, Groby Road, Leicester LE3 9QP, UK. ${ }^{291}$ University Hospitals of Leicester NHS Trust, Leicester General Hospital, Gwendolen Road, Leicester LE5 4PW, UK. ${ }^{292}$ University Hospitals of Leicester NHS Trust, Leicester Royal Infirmary, Infirmary Square, Leicester LE1 5WW, UK. ${ }^{293}$ University Hospitals of Morecambe Bay NHS Foundation Trust, Royal Lancaster Infirmary, Ashton Road, Lancaster LA1 4RP, UK. ${ }^{294}$ University Hospitals of North Midlands NHS Trust, Royal Stoke University Hospital, Newcastle Road, Stoke-on-Trent ST4 6QG, UK. ${ }^{295}$ Walsall Healthcare NHS Trust, Walsall Manor Hospital, Moat Road, Walsall WS2 9PS, UK. ${ }^{296}$ Warrington and Halton Hospitals NHS Foundation Trust, Warrington Hospital, Lovely Lane, Warrington WA5 1QG, UK. ${ }^{297}$ West Hertfordshire Hospitals NHS Trust, Hemel Hempstead General Hospital, Hillfield Road, Hemel Hempstead HP2 4AD, UK. ${ }^{298}$ West Hertfordshire Hospitals NHS Trust, St Albans City Hospital, Waverley Road, St Albans AL3 5PN, UK. ${ }^{299}$ West Hertfordshire Hospitals NHS Trust, Watford General Hospital, Vicarage Road, Watford WD18 OHB, UK. ${ }^{300}$ West Middlesex University NHS Trust, West Middlesex University Hospital, Twickenham Road, Isleworth TW7 6AF.

${ }^{301}$ West Suffolk NHS Foundation Trust, Walnut Tree Hospital, Walnut Tree Lane, Sudbury CO10 1BE, UK. 302 West Suffolk NHS Foundation Trust, West Suffolk Hospital, Hardwick Lane, Bury St Edmunds IP33 2QZ, UK. ${ }^{303}$ Western Sussex Hospitals NHS Foundation Trust, Worthing Hospital, Lyndhurst Road, Worthing BN11 2DH, UK. ${ }^{304}$ Western Sussex Hospitals NHS Foundation Trust, St Richard's Hospital, Spitalfield Lane, Chichester PO19 6SE, UK. 305Weston Area Health NHS Trust, Weston General Hospital, Grange Road, Uphill, Weston super Mare BS23 4TQ, UK. ${ }^{306}$ Whittington Hospital NHS Trust, The Whittington Hospital, Magdala Avenue, London N19 5NF, UK. ${ }^{307}$ Wirral University Teaching Hospital NHS Foundation Trust, Arrowe Park Hospital, Upton CH49 5PE, UK. ${ }^{308}$ Wirral University Teaching Hospital NHS Foundation Trust, Victoria Central Hospital, Mill Lane, Wallasey CH44 5UF, UK. ${ }^{309}$ Worcestershire Acute Hospitals NHS Trust, Alexandra Hospital, Woodrow Drive, Redditch B98 7UB, UK. ${ }^{310}$ Worcestershire Acute Hospitals NHS Trust, Kidderminster Hospital and Treatment Centre, Bewdley Road, Kidderminster DY11 6RJ, UK. ${ }^{311}$ Worcestershire Acute Hospitals NHS Trust, Worcestershire Royal Hospital, Charles Hastings Way, Worcester WR5 1DD, UK. ${ }^{312}$ Wrightington, Wigan And Leigh NHS Trust, Royal Albert Edward Infirmary, Wigan Lane, Wigan WN1 2NN, UK. ${ }^{313}$ Wye Valley NHS Trust, The County Hospital, Stonebow Road, Hereford HR1 2BN, UK. ${ }^{314}$ Yeovil District Hospital NHS Foundation Trust, Yeovil District Hospital, Higher Kingston, Yeovil BA21 4AT, UK. ${ }^{315}$ York Teaching Hospital NHS Foundation Trust, Bridlington Hospital, Bessingby Road, Bridlington YO16 4QP, UK. ${ }^{316}$ York Teaching Hospital NHS Foundation Trust, Scarborough Hospital, Woodlands Drive, Scarborough YO12 6QL, UK. 317 York Teaching Hospital NHS Foundation Trust, The York Hospital, Wigginton Road, York YO31 8HE, UK. ${ }^{318}$ Great Western Hospitals NHS Foundation Trust, Marlborough Road, Swindon, Wiltshire SN3 6BB, UK. 